\title{
Black hole growth and starburst activity at $z=0.6-4$ in the Chandra Deep Field South
}

\section{Host galaxies properties of obscured AGN ${ }^{\star}$}

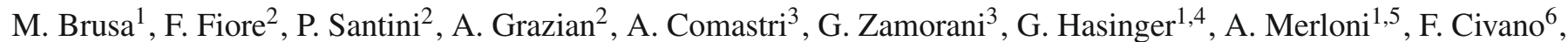 \\ A. Fontana ${ }^{2}$, and V. Mainieri ${ }^{7}$
}

${ }^{1}$ Max-Planck-Institute for extraterrestrial Physik, Giessenbachstrasse, 1, 85748 Garching bei München, Germany e-mail: marcella@mpe.mpg.de

2 INAF - Osservatorio Astronomico di Roma, via Frascati 33, Monteporzio-Catone (Roma) 00040, Italy

INAF - Osservatorio Astronomico di Bologna, via Ranzani 140127 Bologna, Italy

4 Max-Planck-Institute for Plasma Physics, Boltzmannstrasse 2, 85748 Garching bei München, Germany

5 Excellence Cluster Universe, Boltzmannstrasse 2, 85748 Garching bei München, Germany

6 Harvard-Smithsonian Center for Astrophysics, 60 Garden Street, Cambridge, MA 02138, USA

7 European Southern Observatory, Karl-Schwarzschild-str. 2, 85748 Garching bei München, Germany

Received 2 April 2009 / Accepted 22 September 2009

\section{ABSTRACT}

\begin{abstract}
Aims. The co-evolution of host galaxies and the active black holes which reside in their centre is one of the most important topics in modern observational cosmology. Here we present a study of the properties of obscured active galactic nuclei (AGN) detected in the CDFS 1 Ms observation and their host galaxies.

Methods. We limited the analysis to the MUSIC area, for which deep $K$-band observations obtained with ISAAC@ VLT are available, ensuring accurate identifications of the counterparts of the X-ray sources as well as reliable determination of photometric redshifts and galaxy parameters, such as stellar masses and star formation rates. In particular, we: 1) refined the X-ray/infrared/optical association of 179 sources in the MUSIC area detected in the Chandra observation; 2) studied the host galaxies observed and rest frame colors and properties.

Results. We found that X-ray selected $\left(L_{\mathrm{X}} \gtrsim 10^{42} \mathrm{erg} \mathrm{s}^{-1}\right)$ AGN show Spitzer colors consistent with both AGN and starburst dominated infrared continuum; the latter would not have been selected as AGN from infrared diagnostics. The host galaxies of X-ray selected obscured AGN are all massive $\left(M_{*}>10^{10} M_{\odot}\right)$ and, in $50 \%$ of the cases, are also actively forming stars $\left(1 / S S F R<t_{\text {Hubble }}\right)$ in dusty environments. The median $L / L_{\mathrm{Edd}}$ value of the active nucleus is between $2 \%$ and $10 \%$ depending on the assumed $M_{\mathrm{BH}} / M_{*}$ ratio. Finally, we found that the X-ray selected AGN fraction increases with the stellar mass up to a value of $\sim 30 \%$ at $z>1$ and $M_{*}>3 \times$ $10^{11} M_{\odot}$, a fraction significantly higher than in the local Universe for AGN of similar luminosities.
\end{abstract}

Key words. galaxies: active - galaxies: starburst - X-rays: galaxies

\section{Introduction}

Galaxy interactions, and more in general the large scale structure (LSS) galaxy environment, are thought to play a major role in regulating both star-formation and accretion onto nuclear supermassive black holes (SMBHs). This implies that the full understanding of galaxy evolution requires a good knowledge of the SMBH census through cosmic time. In particular, feedback between the central SMBHs in their active phases and the interstellar medium is likely to affect strongly the evolution of their host galaxies.

A short, powerful but highly obscured growth phase of both SMBHs and their host galaxies is predicted by many models for the co-evolution of galaxies and AGN (Silk \& Rees 1998; Fabian 1999; Granato et al. 2004; Di Matteo et al. 2005; Menci et al. 2008). This phase ends when strong AGN winds and shocks heat the interstellar medium, blowing away the dust and gas and inhibiting further star-formation in the AGN host galaxies.

* Tables 1 and 2 are only available in electronic form at http://www . aanda. org
According to this "evolutionary sequence" (e.g. Hopkins et al. 2008), highly obscured AGN should be associated to young galaxies in the process of assembling most of their stellar mass through significant episodes of star formation. On the contrary, unobscured AGN should be associated to galaxies with low or absent episodes of star-formation, given that most of the gas and dust responsible for the star formation has been blown away by the effect of AGN feedback. Many observational evidences (see Alexander et al. 2005; Page et al. 2004; Stevens et al. 2006) and theoretical arguments (Menci et al. 2008, and references therein) in favor of the evolutionary sequence do exist. These results challenge our 20-years old AGN view, in which the differences we see in different classes of sources - especially between "obscured" and "unobscured" ones - are simply due to orientations effects (Antonucci et al. 1985; Antonucci 2003; Urry \& Padovani 1995).

A correct and complete identification of obscured and highly obscured AGN at high redshift is therefore crucial because they may represent the first, still little explored, phase of the common growth of both SMBHs and their host galaxies. 
Moderately and highly obscured AGN at $z \sim 2$, where most of the accretion and star-formation processes are on-going, offer an ideal tool for a direct test of feedback models. In these objects the nuclear light is completely blocked or strongly reduced and does not overshine the galaxy optical and near-infrared light. This gives us the possibility to study host galaxy morphologies, colors and spectral energy distributions without the difficulty of disentangling star-light from nuclear light. We can therefore study galaxy properties during active phases, e.g. when nuclear feedback should be in action.

Obscured AGN at cosmological distances are however usually faint in the observed optical bands, because the UV rest frame is strongly reduced by dust extinction. On the other hand, moderately obscured AGN (or Compton thin, $10^{22}<N_{\mathrm{H}}<$ $10^{24} \mathrm{~cm}^{-2}$ ) are common in X-ray images (Bauer et al. 2004; Comastri \& Brusa 2008, and reference therein), making up $\sim 50 \%$ of the full X-ray population at fluxes $<10^{-14} \mathrm{erg} \mathrm{cm}^{-2} \mathrm{~s}^{-1}$ (Gilli et al. 2007) once the contribution from the normal starforming galaxy population is removed (Ranalli et al. 2005). Compton thick AGN (CT, $N_{\mathrm{H}} \gtrsim 10^{24} \mathrm{~cm}^{-2}$ ) are faint also in the X-ray band, because photoelectric absorption and Compton scattering strongly reduce the X-ray flux up to $>10 \mathrm{keV}$, and over the entire X-ray range if $N_{\mathrm{H}} \gtrsim 10^{25} \mathrm{~cm}^{-2}$. Indeed, only a dozen CT AGN is present in the deepest images of the X-ray sky, the Chandra Deep Fields (Norman et al. 2002; Tozzi et al. 2006; Georgantopoulos et al. 2008).

Identification of the correct counterparts of obscured AGN at cosmological redshift is not trivial. These objects are faint in the optical band, because 1) the intrinsic AGN emission is absorbed by the surrounding material; and 2) the host galaxy light is strongly reduced by cosmological dimming ( $L^{*}$ galaxies at $z=1-2$ have $R \sim 24-25$ ). The probability to find by chance a galaxy of these magnitudes in the Chandra error boxes is not negligible. The identification process is made easier by using deep near infrared images, because the surface density of these sources is smaller than that of optical ones, bringing the probability of finding a galaxy by chance in the Chandra error boxes to comfortably small values. Moreover, the $K$-band flux is more tightly correlated with the X-ray flux than the optical (obscured) one. For this reason, we have reanalyzed the identifications of the Chandra Deep Field South (CDFS) 1 Ms sources (Alexander et al. 2003) in the area covered by sensitive $K$ band and IRAC $3.6 \mu \mathrm{m}$ and $4.5 \mu \mathrm{m}$ observations (the GOODS MUSIC area, Grazian et al. 2006).

The excellent multiband photometry available in this area allows the determination of a reliable photometric redshift for the faint sources not reachable by optical spectroscopy. Once identified the counterparts of the X-ray sources and their redshift, it is possible to proceed to a detailed study of the properties of their host galaxies. We present here a study of the properties of the host galaxies of X-ray obscured AGN, such as their morphology and close environment, optical and infrared rest frame colors, mid infrared to optical spectral energy distributions. Galaxy masses and star-formation rates are derived from the fit of galaxy templates to the optical to near infrared spectral energy distributions. We finally compare the masses and star-formation rates of obscured AGN host galaxies to those of inactive galaxies in the field selected in both optical and infrared bands.

The paper is organized as follows: Sect. 2 presents the CDFS datasets, the X-ray to optical/infrared association and the obscured AGN sample. Section 3 presents the observed frame colors of the obscured AGN sample. Section 4 presents the host galaxies properties (masses and star-formation rates, SFRs) of the sample and our estimates of the fraction of AGN in mass selected samples. Section 5 presents a discussion of the results and Sect. 6 outlines a summary of the most important points. A cosmology with $H_{0}=70 \mathrm{~km} \mathrm{~s}^{-1} \mathrm{Mpc}^{-1}, \Omega_{\mathrm{M}}=0.3, \Omega_{\Lambda}=0.7$ is adopted throughout (Spergel et al. 2003).

\section{Obscured AGN selection in the CDFS}

\subsection{X-ray data: catalogs and multiband identifications}

X-ray data for a total exposure time of $\sim 1 \mathrm{Ms}$ in the GOODS field have been obtained by the CDFS consortium and are publicly available. The source catalog has been published by Giacconi et al. (2002), including the basic X-ray properties in three different X-ray bands (source counts, fluxes, exposure times). Subsequently, Alexander et al. (2003, hereinafter A03) published a re-analysis of the CDFS data that led mainly to an improved astrometry of the X-ray positions with some differences in the number of detected sources at the very faint counts level (see A03 for more details).

Optical identifications for the sources detected by Giacconi et al. (2002), including the position of the candidate counterparts, optical magnitudes and the spectroscopic redshifts from a dedicated VLT campaign, are reported in Szokoly et al. (2004). Photometric redshifts based on good quality optical photometry (VLT and ACS/HST) and deep infrared (ISAAC/VLT) observations are available for all but four CDFS sources without spectroscopic redshift (Zheng et al. 2004; Mainieri et al. 2005), leading to a virtually complete catalog of identified $\mathrm{X}$-ray sources. Using this catalog and redshifts information, Tozzi et al. (2006) derived the rest-frame X-ray properties for the 1 Ms CDFS sources. IRAC photometry, photometric redshifts and radio properties of different subsamples of the CDFS $\mathrm{X}$-ray sources have been published in others works appeared in the literature in the past few years, e.g. Rigby et al. (2005), Alonso-Herrero et al. (2006), Georgantopoulos et al. (2007), Rovilos \& Georgantopoulos (2007) and Tozzi et al. (2009).

These published works rely on the association of the X-ray sources to a candidate optical counterpart. However, given that the optical flux of the X-ray counterparts can be strongly suppressed by dust absorption, an identification process that uses as a prior a catalog generated at a redder wavelength ( $K$-band or IRAC bands) can be more efficient in isolating the correct association to the X-ray source (see, e.g. Brusa et al. 2007). The lower surface density of IRAC sources with respect to faint optical objects is widely used as an argument to indicate that, in case of ambiguous association between two sources, the most likely candidate is the one detected at the brightest IRAC fluxes (whatever the optical flux).

In order to quantify how (and if) the use of catalogs selected in different bands leads to significantly different X-ray to optical associations, we decided to perform again the identification of the Chandra sources, using a new version of the multicolor GOODS-MUSIC sample (GOODS MUlticolor Southern Infrared Catalog; Grazian et al. 2006; De Santis et al. 2007), extracted from the public data of the GOODS-South survey (Giavalisco et al. 2004), and updated as described in Santini et al. (2009). The 15-bands multi-wavelength coverage ranges from 0.35 to $24 \mu \mathrm{m}$, as a result of the combination of images coming from different instruments (2.2ESO, VIMOS/VLT, ACS/HST, ISAAC/VLT, IRAC/Spitzer and MIPS/Spitzer). In the following we will refer to the catalog with full coverage in all bands. Such a catalog covers an area of $143.2 \operatorname{arcmin}^{2}$ and contains 15208 sources. After culling Galactic stars, it contains 14999, extragalactic objects selected in the $z$ band, in the 
$K$ s band and at $4.5 \mu \mathrm{m}$ (see Santini et al. 2009, for further details).

A total of $183 \mathrm{X}$-ray sources in the A03 catalog are found within the GOODS-MUSIC area. Of these, 3 objects are associated with stars (ID \#120,279,289 in A03) and an additional source is most likely a Ultra Lumimous X-ray source (ULX) in a nearby galaxy (ID \#213). Therefore, the number of extragalactic sources (AGN or galaxies) in the MUSIC area is 179, 154 in common with Giacconi et al. (2002) and an additional 25 detected only by $\mathrm{A} 03^{1}$.

167 of the $179 \mathrm{AO} 3$ sources in the MUSIC area are in common with the catalog obtained from the full $2 \mathrm{Ms}$ exposure (Luo et al. 2008). We have visually inspected and carefully checked all the 12 sources missing in Luo et al. (2008). One of these sources, \#225 is most likely a spurious detection on the wings of the Chandra PSF of a brighter source (\#224). Other three sources, \#183, \#215 and \#239, lie in problematic areas, close to a CCD gap and are probably spurious detection in AO3. These four sources are flagged as likely spurious in Table 1 . The other 8 sources present in the AO3 catalog but not in the Luo et al. (2008) catalog are probably real sources, which have not been detected in the full $2 \mathrm{Ms}$ exposure because of variability or a more conservative detection threshold in Luo et al. (2008), or both.

We proceeded for the association of the counterparts to the X-ray sources as follows. First, we used a statistical method (the "likelihood ratio technique", following the approach described by Brusa et al. (2007) to isolate the most likely $z$-band, $K$-band and IRAC counterparts from the $z-, K$ - and 4.5 micron selected catalogs in the MUSIC area. The method calculates the probability that a source is the correct association by weighing the information on the X-ray to optical distance, the surface density of (possible) false coincidence background objects and the brightness of the chosen counterpart. With this method we were able to isolate the most obvious associations, and, at the same time to pick up the objects with 2 or more different counterparts with comparable likelihood in the same or different bands $(<10 \%$ of the entire sample). Then, we visually inspected all the proposed 179 associations in order to resolve possible ambiguous cases. The result of our work is summarized in Table 1. For each X-ray source we report the X-ray identificator from A03 (AID, Col. 1), the X-ray identificator from G02/S04 (XID, Col. 2), the MUSIC identificator (OID, Col. 3), the positions of the proposed counterpart (Cols. 4,5$)$, the distance of the chosen counterpart from the X-ray centroid (Col. 6), a flag for the identification (Col. 7), the observed X-ray fluxes in the hard $(2-10 \mathrm{keV})$ and soft $(0.5-2 \mathrm{keV})$ bands (Cols. 8 and 9), the redshift of the source (Col. 10, see next section) and the rest frame 2-10 keV luminosity (Col. 11). The X-ray fluxes (in erg $\mathrm{cm}^{-2} \mathrm{~s}^{-1}$ ) have been derived from the counts reported in A03, using the following conversion factors:

$F(2-10 \mathrm{keV})=2.8 \times 10^{-11}$ Counts $(2-8 \mathrm{keV})$

$F(0.5-2 \mathrm{keV})=5.6 \times 10^{-12}$ Counts $(0.5-2 \mathrm{keV}$

for a power law spectrum with energy index equal to 0.4 , i.e. the average value for deep fields sources (see, e.g., Tozzi et al. $2001)^{2}$.

\footnotetext{
1 We do not discuss here the 10 sources in the MUSIC area present only in Giacconi et al. (2002).

2 We haven't used the fluxes tabulated by A03 because they have been calculated with different (source-by-source) energy indeces and make it difficult to correct for the absorption and derive rest-frame, unobscured luminosity (see 2.2).
}

Of the 154 published counterparts (first and second part of the table, mostly from Szokoly et al. 2004, but see also notes in Table 1), we were able to confirm 144 X-ray to optical associations $(93.5 \%$, sources with flag $=0)$, we changed 9 X-ray to optical associations $(5.8 \%$, flag $=1)$ ), while for the remaining case (AID 186) we were not able to assign a reliable IRAC or optical counterpart $(0.6 \%$, i.e. neither to confirm the published counterpart nor to propose a new counterpart). The fraction of previously misidentified sources rises up to $15 \%$ when the optically faint $(z>24)$ sample is considered $(9 / 61)$.

Figure 1 shows the Chandra, ACS ( $z$-band), $K$ and IRAC4.5 micron cutouts for the 9 sources we identify with a counterpart different from that proposed in the literature (see again notes in Table 1). The cyan circle marks the new counterpart, while the yellow circle marks the old counterpart. In almost all the cases the new proposed counterpart is absent in the optical images.

For the remaining 25 sources detected only in A03, we were able to unambiguously identify the counterpart for 23 of them. The optical counterparts of these 23 sources are reported in the third part of Table 1 .

We computed the rest frame, absorption corrected 2-10 keV luminosities from the $2-10 \mathrm{keV}$ fluxes, when available, and from the $0.5-2 \mathrm{keV}$ fluxes in the remaining cases. We assumed a power law spectrum with $\alpha_{E}=0.8$ reduced at low energy by rest frame photoelectric absorption. We used the $N_{\mathrm{H}}$ values reported by Tozzi et al. (2006) for the sources with redshift consistent between this and the previous work ( $\sim 68 \%$ of the sources have $|\Delta z /(1+z)|<0.2$, see also next section). For the remaining $32 \%$ of the sources we evaluated $N_{\mathrm{H}}$ from the hardness ratio between the hard and soft bands.

\subsection{Optical+Infrared photometry and photometric redshifts}

Table 2 shows the compilation of optical $b, v, i, z$ bands (Cols. 2-5) from GOODS data (Santini et al. 2009), infrared $J, H, K$ (Cols. 6-8) from ESO/VLT, IRAC and MIPS (Cols. 9-13) photometry. Of the 179 X-ray sources, 174 are detected in the $z$-band, 172 in the $4.5 \mu \mathrm{m}$ IRAC channel and 133 in the $24 \mu \mathrm{m}$ MIPS survey.

The spectroscopic or photometric redshifts available for the proposed optical counterparts of the 179 CDFS-GOODS sources are listed in Col. 10 of Table 1. When a spectroscopic redshift is present, the relative source catalog is reported, as follows: (1) CXO-CDFS (S04); (2) K20 (Mignoli et al. 2005); (3) GOODS (Vanzella et al. 2005, 2006, 2008; Popesso et al. 2008); (4) COMBO-17 (Wolf et al. 2003); (5) VVDS (Le Fevre et al. 2004); (6) other (Daddi et al. 2005; Cristiani et al. 2000). Otherwise, the redshift is from SED fitting (Santini et al. 2009) and is reported with its $1 \sigma$ confidence interval. We have spectroscopic or photometric redshifts for all but one (AID 282) of the identified X-ray sources. However, in eleven cases, only a lower limit on the photometric redshift solution is available from the SED fitting, i.e. at higher redshifts the $\chi^{2}$ curve is relatively flat and an upper limit on the basis of a $\Delta \chi^{2}$ could not be determined.

Figure 2 shows the redshift distribution of the entire sample (open black histogram) and the distributions derived for the spectroscopic and photometric sub-samples (blue and red histograms, respectively). About half of the redshifts at $z>1$ come from the photometric sample. For about $25 \%$ of the sources the redshifts listed in Table 2 are different (i.e. not consistent within the errors) from the ones published in previous works (as compiled by Zheng et al. 2004; Tozzi et al. 2006), for the following reasons: 1) for 9 objects the counterparts have changed from the ones reported in the literature (see previous section); 2) for about 


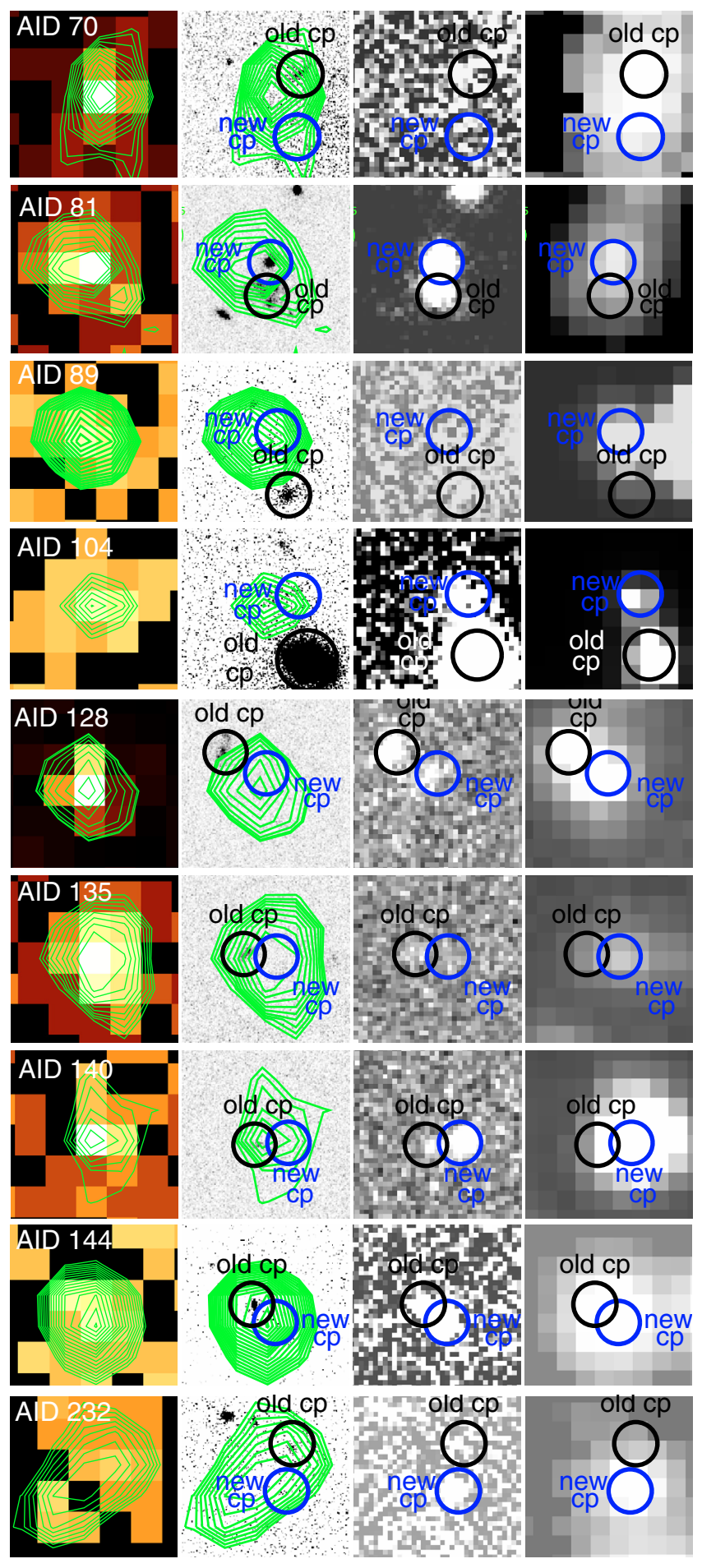

Fig. 1. Chandra, ACS ( $z$-band), $K$-band and IRAC-4.5 micron cutouts $\left(5^{\prime \prime} \times 5^{\prime \prime}\right)$ of the 9 sources identified with a counterpart different from that proposed in the literature. The blue circle marks the new counterpart, while the black circle marks the old counterpart. Smoothed countours from the X-ray image are superimposed on the ACS image.

30 sources the availability of longer wavelength (IRAC) data increases the reliability of the photometric redshifts. The photometric redshifts for the sources in the MUSIC sample are instead consistent with a new analysis of the sources in the 2 Ms CDFS (Luo et al. 2008) and the Extended Chandra Deep Field South (E-CDFS, Lehmer et al. 2005) surveys obtained by Rafferty et al. (2009, in preparation).

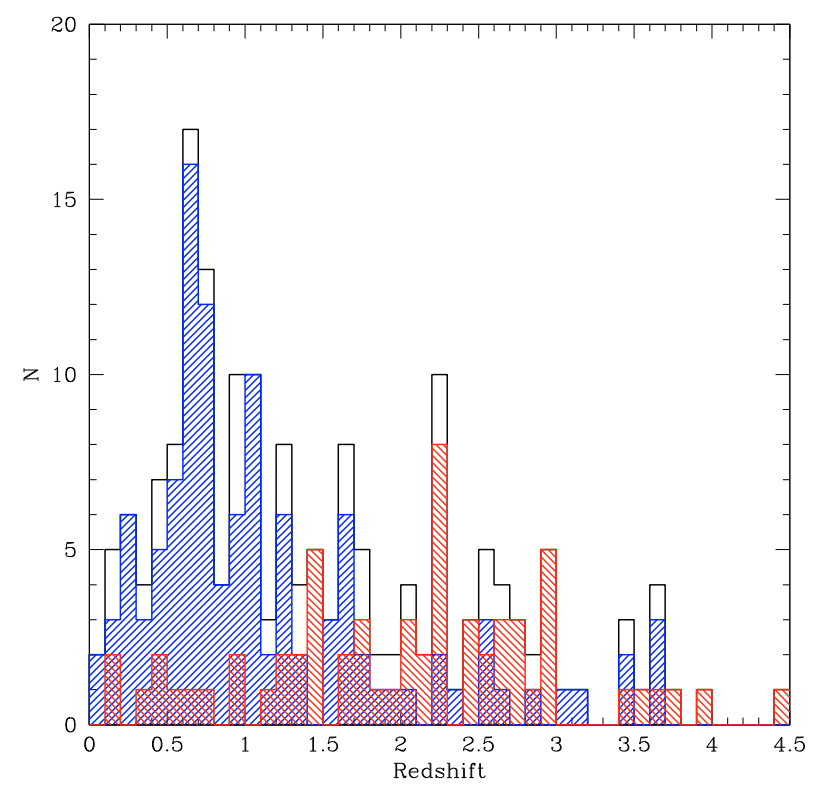

Fig. 2. Redshift distribution of the X-ray sources. Open histogram: all sources; right diagonal (blue) shaded histogram: spectroscopic sample; left diagonal (red) shaded histogram: photometric sample.

\subsection{Obscured AGN sample}

We extracted from our sample of 179 sources, the sample of "obscured" AGN defined as outlined below. First, we considered all the sources with rest frame $\log L(2-10 \mathrm{keV})>41.8$ in order to minimize the contribution of non-AGN objects among the X-ray selected sample. Indeed, following Ranalli et al. (2003), the observed X-ray emission can be due to stellar processes only in objects with SFR larger than $\sim 100 M_{\odot} \mathrm{yr}^{-1}$ (see also Sects. 4.2 and 4.4 for further discussion on the luminosity threshold). We also removed one source that shows extended X-ray emission, and the three sources without a unique/secure identification (see Sect. 2.1). We were left with 142 sources. Secondly, we isolated all the 19 sources spectroscopically identified as Broad Line (BL) AGN in Szokoly et al. (2004), and additional 7 objects still spectroscopically unidentified but with a $s g$ parameter (stellarity, from SExtractor, Bertin \& Arnouts 1996) larger than 0.9. We named these 26 sources as BL/pointlike sample. Following Silverman et al. (2008), we computed the ratio of the flux in the $z_{850}$-band in two fixed apertures (a small aperture with a radius of 3 pixels $\left(0.09^{\prime \prime}\right)$ and a larger aperture with a radius of 25 pixels $\left.\left(0.75^{\prime \prime}\right)\right)$. This quantity is similar to the inverse of the concentration index (Abraham et al. 1994). Fluxes were determined by extracting counts from background-subtracted cutouts of ACS images centered aroud the position of the X-ray sources. Figure 3 shows the distribution of the $z$-band counts ratio for the BL/pointlike sample (blue histogram), and the remaining sources (116 objects, red histogram). All the objects with ratio values $>0.3$ are from the BL/pointlike sample, as expected in sources for which the pointlike and/or nuclear emission dominates overall the host-galaxy light. The contribution of pointlike/unobscured AGN at ratio values $<0.3$ is $\sim 10 \%$ (being zero in the two lowest ratio bins, $0-0.1$ ), therefore we expect a negligible contamination from this class of sources in the sample with ratio $<0.3$. We call "obscured" AGN the 116 objects with the ratio of $z$-band counts $<0.3$ and not classified as BL AGN or pointlike in the ACS images. Ninety of these "obscured" AGN $(\sim 75 \%)$ are also detected at MIPS $24 \mu \mathrm{m}$. 


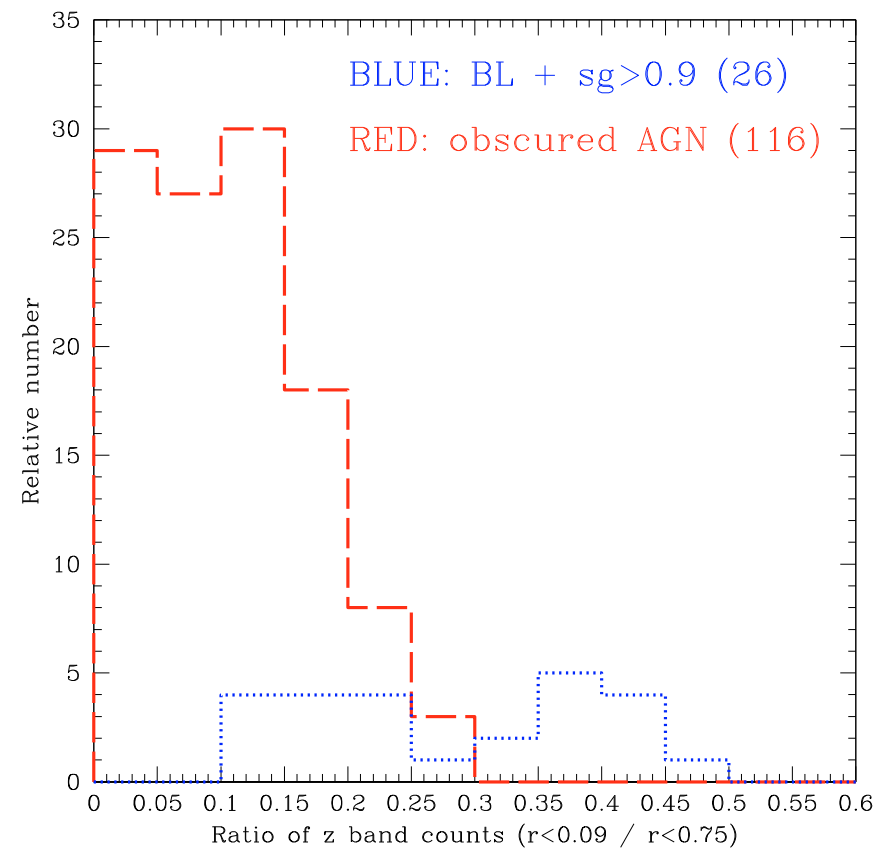

Fig. 3. Distribution of the $z$-band count ratio for the "obscured" AGN sample (116 objects, red long dashed histogram) and the $\mathrm{BL} /$ pointlike sample (26 objects, blue dotted histogram).

X-ray column densities from the 1 Ms sources in the CDFS derived from an accurate spectral fitting analysis have been published by Tozzi et al. (2006). As discussed in the previous section, a fraction of the X-ray to optical associations changed from the Tozzi et al. (2006) analysis to the present analysis (9 sources), and in addition some more robust photometric redshifts have been obtained using the Spitzer photometry. Among the sixty-nine sources in the "obscured" AGN sample in common with the Tozzi et al. sample ${ }^{3}$ and with a $|\Delta z /(1+z)|<0.2$ from the redshift values reported in Tozzi et al. (2006), fiftyfour $(\sim 80 \%)$ show X-ray absorption in excess of $10^{22} \mathrm{~cm}^{-2}$, further suggesting that our sample is representative of the $\mathrm{X}$-ray obscured AGN population.

We visually inspected all the sources in the $z$-band ACS images and divided them in three main classes: isolated sources (35), interacting/disturbed/clumpy galaxies (53) and objects too faint to tell something on the morphology (26). All of these 26 sources populate the first bins in the histogram in Fig. 3.

Figure 4 shows four examples of obscured AGN counterparts with disturbed morphology or interacting systems.

\subsection{Host galaxy properties}

For the host galaxies of the obscured AGN we retrieved from the GOODS-MUSIC catalog the rest frame $U-V$ colors, the absolute $V$ and $K$-band magnitudes, the SFRs and the stellar masses $M_{*}$ derived from the SED fitting. More specifically, stellar masses are derived from the comparison of SED and libraries of synthetic spectra, assuming a Salpeter (1955) Initial Mass Function (IMF) and simple stellar population (SPP) models (Bruzual \& Charlot 2003), as described at length in Fontana et al. (2006). SFRs have been estimated from SED fitting, using Bruzual \& Charlot (2003) synthetic models and fitting the

\footnotetext{
${ }^{3}$ We recall that our sample contains 16 sources detected only in the A03 sample and not present at all in the Tozzi et al. (2006) spectral analysis.
}

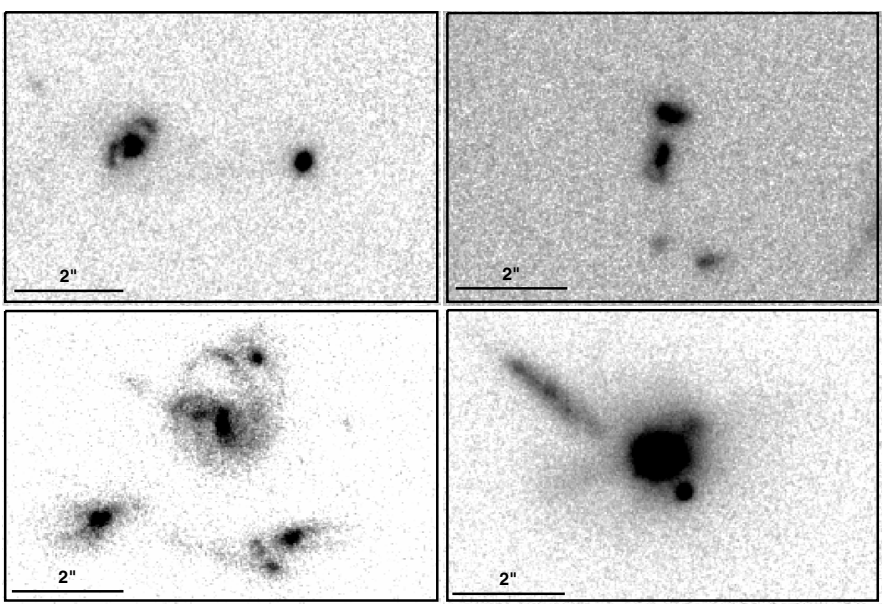

Fig. 4. ACS (z-band) images of obscured AGN counterparts with disturbed morphology or interacting systems (AID 88, 107, 226 and 84).

UV rest frame photometric bands. We parametrize the star formation histories with a variety of exponentially declining laws (with timescales ranging from 0.1 to $15 \mathrm{Gyr}$ ), metallicities (from $Z=0.02 Z \odot$ to $Z=2.5 Z \odot)$ and dust extinctions $(0<E(B-V)<$ 1.1 , with a Calzetti or Small Magellanic extinction curve). These SFR estimates have been compared with the ones derived from the observed IR (MIPS) emission, following the approach presented by Papovich et al. (2007): despite the majority of galaxies are assigned a consistent SFR (with overall median ratio around unity, see details in Santini et al. 2009), in the following, we will use the SFR from the SED fitting for all the obscured AGN in our sample, in order to minimize the possible AGN contribution in the computation of SFRs from MIPS data in AGN samples (see Daddi et al. 2007a).

As a word of caution, we note here that the derivation of host galaxy properties is affected by both statistical and systematics undertainties.

Statistical errors for both masses and SFR have been calculated from the $1 \sigma$ convidence level in each parameter estimate by scanning the $\chi^{2}$ levels, allowing the redshift to change within the errors in case of objects with photometric redshifts ${ }^{4}$ (see Fontana et al. 2006; Santini et al. 2009).

The masses and SFR estimates are, however, limited by the systematics related to model degeneracy and, in particular, the ones associated to the adopted IMF, the SSP, the metallicity and the star formation history (see Santini et al. 2009; and the discussion in Ilbert et al. 2009). The largest, systematic, uncertainty on the estimate of the stellar mass is the assumption on the IMF. The masses obtained using a Chabrier IMF are on average 0.24 dex lower than those obtained adopting a Salpeter IMF, and this effect is totally independent from the range of redshift and mass probed (Salimbeni et al. 2009). The uncertainties related to the adopted SSP models (Bruzual \& Charlot 2003; Charlot \& Bruzual 2007; Maraston 2005) to construct libraries of synthetic spectra, are also of the order of $\Delta(\log M)=0.2-0.3$, but the effect is less evident in the high-mass part and at high- $z$. (see Salimbeni et al. 2009, for a proper discussion on these effects in the GOODS-MUSIC sample).

Similar systematics effects are present also in the SFR estimates, and are expected to be of the order of

\footnotetext{
4 We note here that photo- $z$ accuracy, in general, is not the leading source of error in deriving the galaxies and AGN parameters (see also discussion in Ilbert et al. 2009).
} 

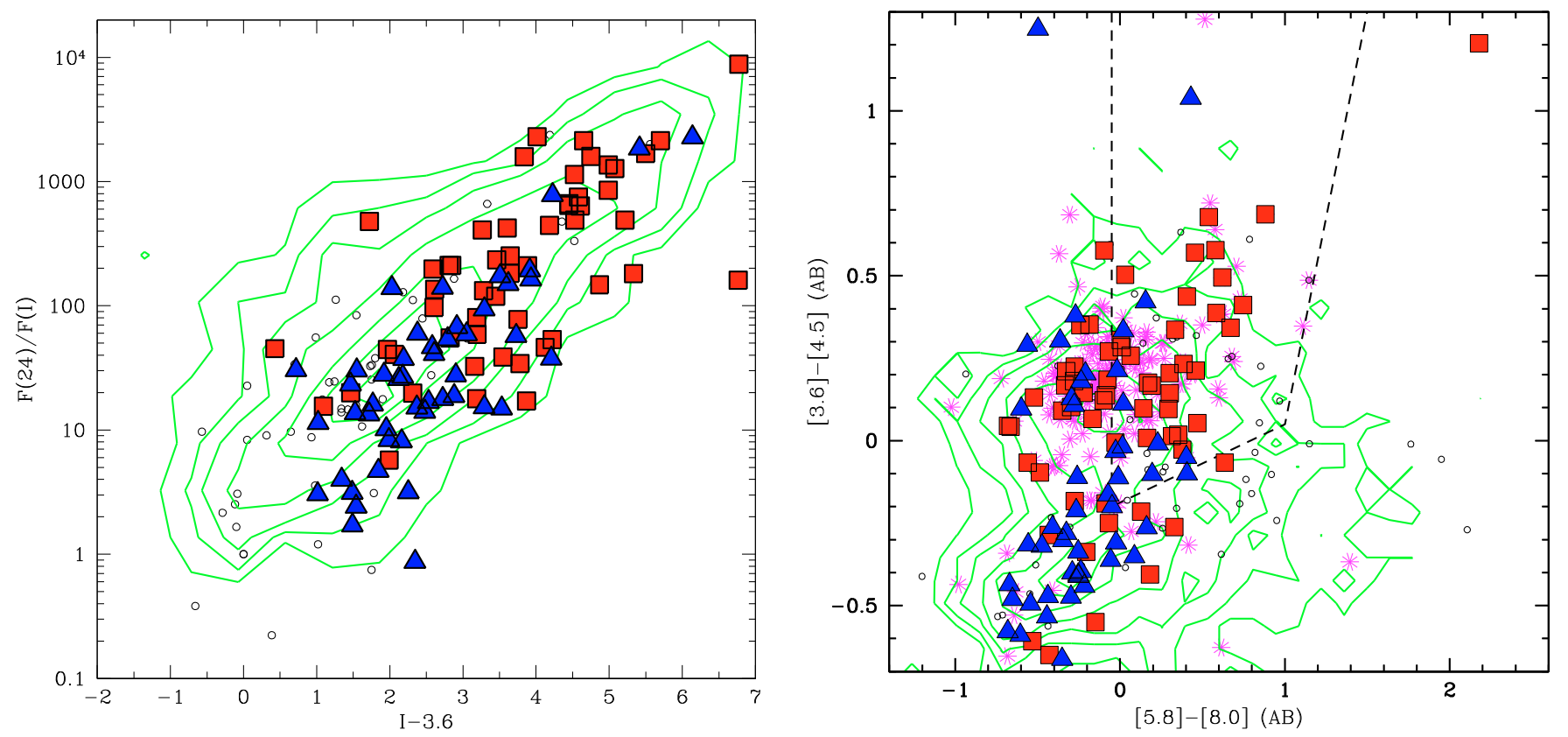

Fig. 5. Left panel: $24 \mu \mathrm{m}$ to optical ( $I$-band) flux ratio vs. the $I-[3.6]$ color for the obscured AGN in our sample. The sizes and shape (colors) of the symbols reflect the AGN X-ray luminosity: (blue) small triangles are objects with $\log L_{X}<43$, (red) large squares objects with $\log L_{X}>43$. Open circles mark all the other X-ray sources (both BL/pointlike AGN and sources with $\log L(\mathrm{X})<41.8$ ). The green contours are the isodensities of the $24 \mu \mathrm{m}$ selected population. Right panel: IRAC color-color: [3.6]-[4.5] color vs. [5.8]-[8.0] color. The dotted lines isolate a wedge for the selection of bright, optically unobscured quasars (see Stern et al. 2005). The color-code is the same as in left panel. The magenta asterisks are objects in the MIPS sample selected on the basis of their high $24 \mu \mathrm{m}$ to optical flux ratio and extremely red colors $(R-K>4.5)$.

$\Delta(\log S F R) \sim 0.2-0.4$ (see, e.g., discussion in Ferreras et al. 2005 , for the variation due to different IMF).

Since most of these effects are systematics and since we are interested at general trends over several decades of mass and not in the detailed description of the mass of the host galaxies of each X-ray source, we are confident that the overall results discussed in the rest of the paper remain unchanged.

\section{Observed frame colors}

A proper classification of source nature (AGN vs. starburst vs. normal evolved galaxy) should ideally be obtained via a complete analysis of its emission over the electromagnetic spectrum, using both spectroscopic (e.g. emission line widths and ratios) and photometric (all-wavelengths sampled SED) observables. However, in reality, a complete source characterization is not obtainable, even in fields where the best and deepest imaging and spectroscopic campaigns have been performed, such as the GOODS/MUSIC field. Partial, but still reliable, information (at least in a statistical sense) can be obtained through the analysis of the emission in bands where differences between nuclear and star formation emission are emphasized, comparing the observed SEDs with galaxy templates over a range as broad as possible. In particular, the combination of observed-frame mid-infrared, near infrared and optical flux ratios has been exploited recently in the literature to isolate obscured AGN (see, e.g., Martinez-Sansigre et al. 2005, 2006; Fiore et al. 2008; Donley et al. 2008; Dey et al. 2008).

Figure 5, left panel shows the $24 \mu \mathrm{m}$ to optical (I-band) flux ratio vs. the $I-[3.6]$ color for the X-ray sources in our sample. The green contours show the isodensities of the field objects in the MIPS selected sample. The sizes and shape (colors) of the symbols reflect the AGN 2-10 keV luminosity: (blue) small triangles are objects with $\log L_{\mathrm{X}}<43$, (red) large squares objects with $\log L_{X}>43$. Open circles mark all the other X-ray sources (both BL/pointlike AGN and sources with $\log L(\mathrm{X})<$ 41.8). The well-established correlation between the MIR/O color and the total source luminosity traced in this case by the X-ray emission (see Fiore et al. 2008) is also present in this plot, where the most luminous sources (red squares) cluster at average values of MIR/O ratio higher than those of less luminous sources (blue triangles), and on average towards redder optical to near infrared colors.

The right panel of Fig. 5 shows an IRAC color-color diagram where the [3.6]-[4.5] color is plotted against the [5.8]-[8.0] color. The dotted lines isolate a wedge for the selection of bright, optically unobscured quasars, originally proposed in Stern et al. (2005). The sizes and shapes (colors) of the symbols reflect the AGN X-ray luminosity, as in the left panel of Fig. 5. Open small symbols are unobscured AGN and all the other X-ray sources. While the most luminous "obscured" AGN occupy the region in the color-color diagram expected from a power-law SED, i.e. within the wedge marked by the dashed lines, the moderate luminous "obscured" AGN population mainly clusters in two regions with roughly the same [5.8]-[8.0] color but with a bimodal distribution in the [3.6]-[4.5] color (see also results presented by Cardamone et al. 2008). About half of the moderate luminous obscured AGN in the sample are clustered around [3.6]-[4.5] $\sim-0.25$. Most of these objects are in the redshift range $z=0.6-0.8$ (i.e. in the structures present in the CDFS field, see Gilli et al. 2005; Silverman et al. 2008). In this region also fall the $60 \%(4 / 7)$ of the radio detected sources in the obscured AGN sample, extracted from the work by Tozzi et al. (2009, see also the results presented by Hickox et al. 2009). The other half of the obscured AGN sample clusters at redder [3.6]-[4.5] colors 


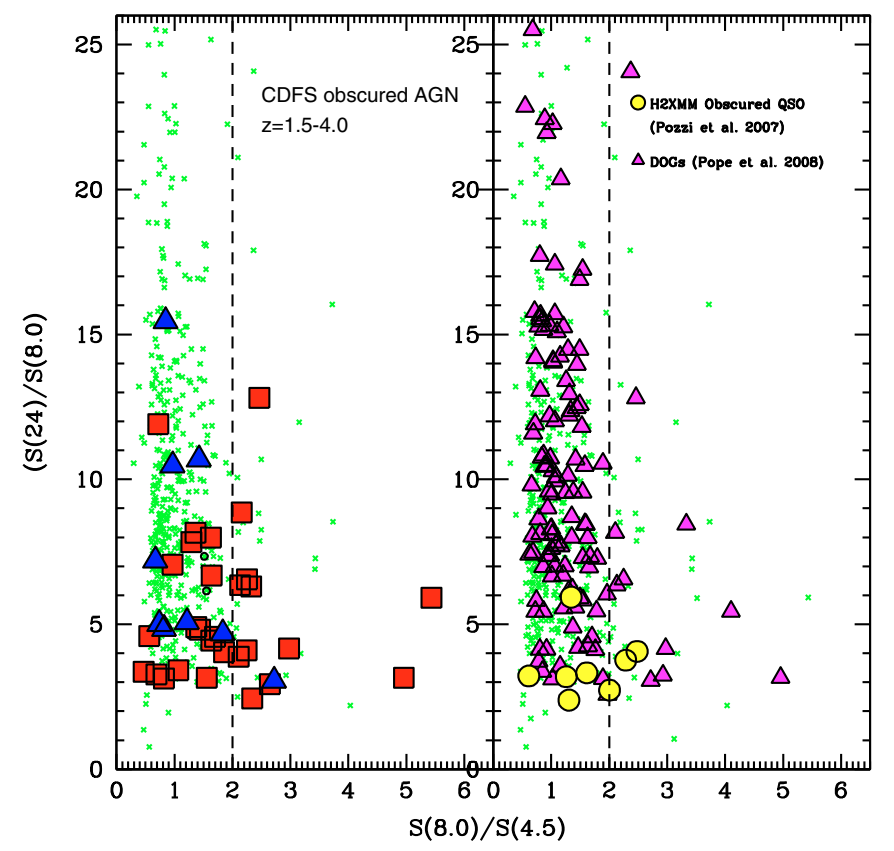

Fig. 6. Spitzer color color diagram used to isolate star-formation and AGN dominated high- $z$ galaxies (Pope et al. 2008). The small green points represent the distribution of field galaxies in the $24 \mu \mathrm{m}$ MUSIC sample in the same redshift range. Objects with $S(8.0) / S(4.5)<2$ are classified "star-formation dominated" by Pope et al. (2008). In the left panel we superimpose to the MUSIC/MIPS population the objects in our obscured AGN sample. The color code for the large symbols is the same as in Fig. 5. Only objects at $z=1.5-4$ have been included. In the right panel other samples of high- $z$ obscured AGN are plotted, as labeled.

$(\sim 0.25)$, where both star-forming and normal galaxies at $z \sim 1-3$ are expected. The isodensity curves from the GOODS-MUSIC sample (Santini et al. 2009), overlaid as green contours in the plot, show the same bimodal distribution as observed for the $\mathrm{X}$-ray sources.

In Fig. 5, right panel, we also plot as magenta asterisks the population of the most obscured, possibly Compton Thick AGN at $z \sim 2$ discussed by Fiore et al. (2008) and selected on the basis of their high $24 \mu \mathrm{m}$ to optical flux ratio and extremely red colors $(R-K>4.5$; see also Daddi et al. 2007a; Dey et al. 2008). These $\mathrm{X}$-ray undetected, Compton Thick AGN candidates occupy the same region in which X-ray detected, $z>1$ obscured AGN lie (see also Georgantopoulos et al. 2008).

Figure 6 shows the combined MIPS-IRAC color-color diagram introduced by Pope et al. (2008; see also Ivison et al. 2004) to distinguish between AGN and star-formation dominated sources in high- $z(z>1.5)$ infrared selected sources, where the $24 \mu \mathrm{m}$ to $8.0 \mu \mathrm{m}$ flux ratio is plotted against the $8.0 \mu \mathrm{m}$ to $4.5 \mu \mathrm{m}$ flux ratio. Indeed, at $z>1.5$, the observed $24 \mu \mathrm{m}$ emission samples the rest frame $<10 \mu \mathrm{m}$ emission, where strong starburst PAH lines are commonly present and can boost the measured IR flux, giving a higher $24 \mu \mathrm{m}$ to $8.0 \mu \mathrm{m}$ flux ratio. Similarly, the $8.0 \mu \mathrm{m}$ to $4.5 \mu \mathrm{m}$ flux ratio is a measure of the rest-frame near infrared continuum slope. Pope et al. (2008) proposed that dust obscured galaxies with large values of the $8.0 \mu \mathrm{m}$ to $4.5 \mu \mathrm{m}$ flux ratio ( $>2$; dotted line in Fig. 6) are AGN dominated sources, according to the presence of AGN continuum in Spitzer IRS spectra, while objects with lower values of the $8.0 \mu \mathrm{m}$ to $4.5 \mu \mathrm{m}$ flux ratio $(<2)$ are "star-formation dominated", in agreement with the fact that they span a wide range of $24 \mu \mathrm{m}$ to $8.0 \mu \mathrm{m}$ flux ratio. In both panels of this figure, we plot the field population extracted from the MUSIC sample as small crosses. In the left panel, we report as squares the CDFS obscured AGN with a MIPS $24 \mu \mathrm{m}$ detection, and in the redshift range $z=1.5-4$ (38 objects in total). In the right panel, we mark as triangles the sources satisfying, among the underlying population, the dust obscured galaxies selection used by Pope et al. (MIPS/O ratio larger than 1000 and $R-K>4.5$, see also Fiore et al. 2008; Dey et al. 2008) and as circles the X-ray selected obscured QSO from the Hellas2XMM survey for which deep Spitzer imaging has been obtained (Pozzi et al. 2007).

According to the classification introduced by Pope et al. (2008), the subsample of X-ray selected, "obscured" AGN with MIPS detection show Spitzer colors consistent with both an AGN dominated continuum and a starburst dominated continuum in the mid-infrared. Actually, a large fraction of the most X-ray luminous AGN lie in the Pope et al. (2008) "starformation dominated" part of the diagram, similarly to the X-ray selected obscured quasars from the Hellas2XMM survey. In conclusion, for at least half of the obscured AGN with MIPS detection in our sample, the accretion activity is revealed thanks to the presence of the X-ray emission and not by a large $8.0 \mu \mathrm{m}$ to $4.5 \mu \mathrm{m}$ flux ratio. Moreover, about half (13/25) of the sources classified as AGN in the "starburst" part of the diagram have $24 \mu \mathrm{m}$ to $8.0 \mu \mathrm{m}$ flux ratio lower than 5 , while in the original Pope et al. diagram this region is almost empty.

\section{Rest-frame colors: host galaxy properties}

\subsection{Reddening}

The left panel of Fig. 7 shows the $U-V$ (rest) vs. the absolute $V$-band magnitude for X-ray selected "obscured" AGN (squares) and the underlying optically selected galaxy population (contours) in the redshift interval $z=0.6-4.0$. The sizes and colors of the symbols reflect the AGN X-ray luminosity (as in Figs. 5 and 6). X-ray selected, obscured AGN show a $(U-V)$ rest color redder than the average color of the optically selected galaxy population and are hosted preferentially by luminous $(M V<-20)$, red $(U-V>0.5)$ galaxies, populating mostly the red sequence and the green valley of the bimodal color-magnitude distribution observed for field objects. We do not find any trend with the X-ray luminosity: the $U-V$ color does not depend on the strength of the X-ray emission. This result is in agreement with previous findings reported in the literature (Nandra et al. 2007; Rovilos \& Georgantoupolos 2007; Silverman et al. 2008; Hasinger 2008); in the next section we will further discuss it and its consequences for our fully understanding of AGN hosts properties.

The right panel of Fig. 7 shows the [3.6]-[4.5] color vs. the $U-V$ (rest frame) color for the sample of obscured AGN (squares) and the underlying optically selected galaxy population (contours), in three different redshift bins: $z=0.6-1.0$, $z=1-2$, and $z=2-4$ from left to right, respectively. The upper panels show the comparison of X-ray selected obscured sources with the optically-selected ( $z$-band) galaxy population; the lower panels show the comparison for the subsample of objects detected also at $24 \mu \mathrm{m}$.

We do not find a significant evolution in the $(U-V)$ rest frame color with redshift for both the overall galaxy population and the obscured AGN sample. Instead, the average value of the [3.6]-[4.5] color increases with redshift and the average [3.6]-[4.5] color of the obscured AGN is consistent with that of the underlying galaxy population (see also Fig. 5). The only difference among the field and obscured AGN population is, 

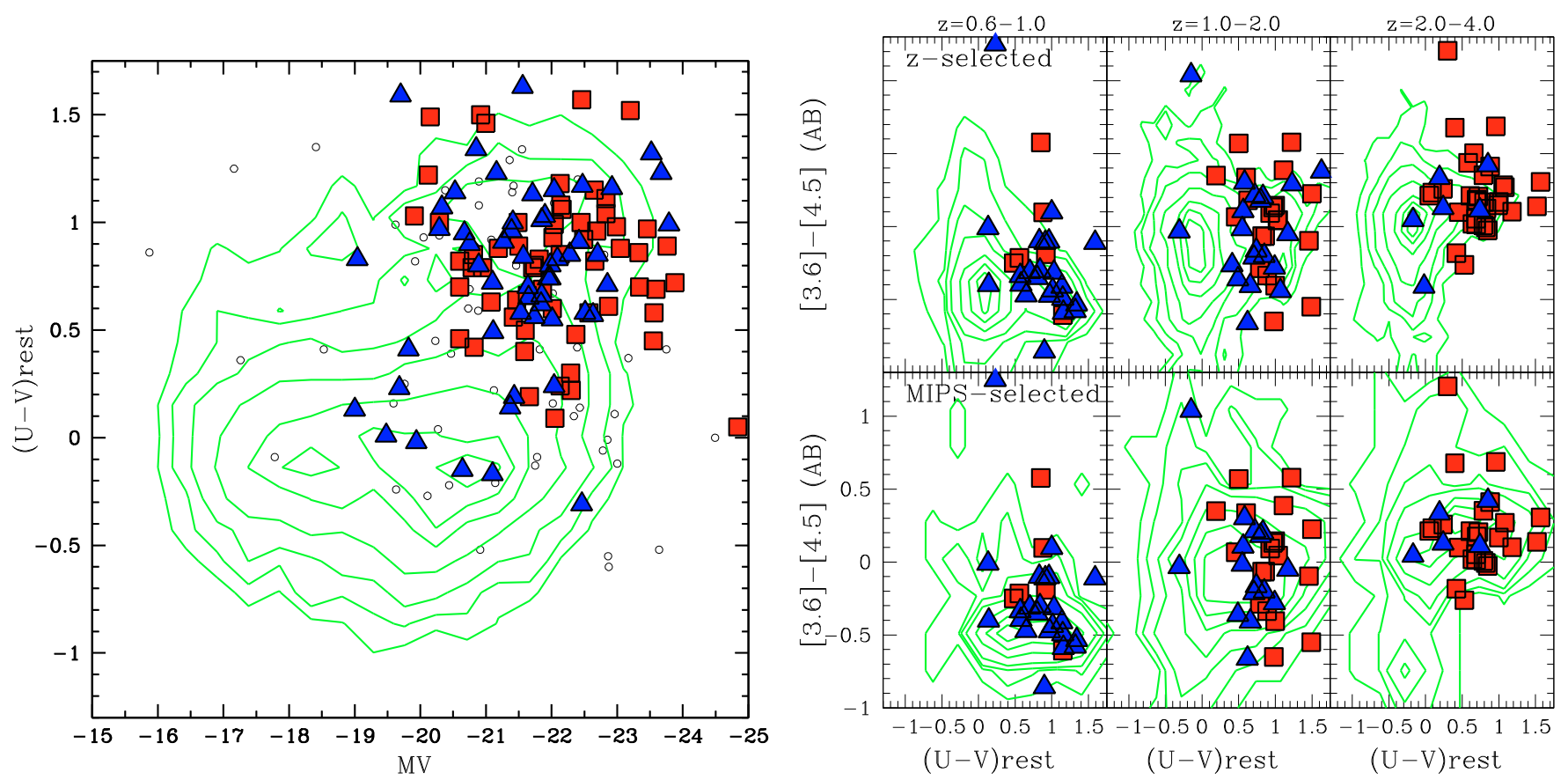

Fig. 7. Left panel: $U-V$ (rest frame) vs. the absolute $V$-band magnitude for X-ray obscured AGN (squares) and the underlying optically selected galaxy population (contours). The sizes and colors of the symbols reflect the AGN X-ray luminosity (as in Figs. 5 and 6). Right panel: [3.6]-[4.5] color vs. the $U-V$ (rest frame) for obscured X-ray sources (squares) and the underlying optically selected galaxy population (contours). The upper panels show the comparison of the X-ray selected sources with the optically-selected (z-band) galaxy population in three different redshift bins: $z=0.6-1.0, z=1-2$, and $z=2.4$ from left to right, respectively. The lower panels show the comparison for the subsample of objects detected also at 24 micron, in the same redshift bins. The sizes and colors of the symbols reflect the AGN X-ray luminosity as in Figs. 5 and 6. Unobscured AGN are not shown here.

therefore, the fact that obscured AGN are redder, in $U-V$ color, than optically selected sources (as outlined in the left panel), while sharing the same [3.6]-[4.5] color. This is probably due to the fact that the observed [3.6]-[4.5] color strongly correlates with redshift and it is not related to any intrinsic property of the sources (e.g. star formation or AGN activity). If we consider the MIPS selected sample (lower panels) the X-ray selected, obscured AGN closely follow the distributions of the underlying star-forming population with redder $U-V$ rest frame colors than in the $z$-selected sample.

Figure 8 (left panel), shows the $U-V$ (rest frame) vs. the absolute $K$-band magnitude for the obscured AGN in our sample (squares) and the isodensity contours of the galaxy population (green curves), in the same redshift bins as in Fig. 7, and for both the optically ( $z$-band) and MIPS selected samples.

At all redshifts, most of the obscured AGN host galaxies show absolute magnitude brighter than $M_{K}<-23$, much brighter than that of the bulk of the optically selected population (upper panel): the large majority of optically selected galaxies cluster around lower values of $M_{K}$ and bluer values of the $(U-V)$ rest frame colors.

The distribution of sources in the $U-V$ vs. $M_{K}$ plane is different when the MIPS selected sample is considered: obscured AGN hosts share the same $U-V$ colors of the mid infrared selected galaxy population. In this case, a color bimodality is not observed at any redshift, and the $U-V$ color correlates smoothly with the galaxy mass (also see Aussel et al. 2009, in prep.). AGN hosts are found preferably at the peak of the galaxy isodensity contours in Fig. 8 lower panels. In particular, the comparison of the upper and lower panels of Fig. 8 suggests a scenario in which star forming galaxies hosted in low mass systems are not particularly dust obscured and therefore the reprocessed emission is not revealed at IR bands (at least at the limiting fluxes of the $24 \mu \mathrm{m}$ GOODS survey), consistent with the results by Daddi et al. (2007b).

Given that $M_{K}$ is a good tracer of the stellar mass of the galaxies, this suggests that obscured AGN are preferentially hosted in high-mass systems. This is shown in the right panel of Fig. 8, where the best fit $\log M_{*}$ is plotted against the redshift (see also Alonso-Herrero et al. 2008) for both the field population from the MUSIC catalog (crosses) and the obscured AGN (red circles). As discussed in Sect. 2.4, the determination of the stellar mass suffers from severe systematic uncertainties. However, these uncertainties are present for both the AGN and the underlying galaxy population, with the net results of a likely shift in the $Y$-axis $\left(\Delta \log M_{*}=-0.3\right)$.

\subsection{Star formation activity}

The black open histogram in Fig. 9 left panel, shows a normalized distribution of the SFR (see Sect. 2.4) for both the entire MUSIC catalog (upper panel) and the MIPS selected sample (lower panel). The red filled histogram shows the distribution of SFR for the "obscured" AGN in our sample. In all cases the SFR have been restricted to objects in the redshift interval $z=0.6-4$, and in the stellar mass range $M_{*}=10^{10}-10^{12} M_{\odot}$. We chose this stellar mass range because most of the obscured AGN in our sample have masses larger than this value (see Fig. 8 right panel) and the completeness of both the entire MUSIC catalog and the MIPS selected sub-sample have been studied in details (see discussion in Fontana et al. 2009). The total sample considered here includes 103 obscured AGN (82 in the MIPS detected subsample). 

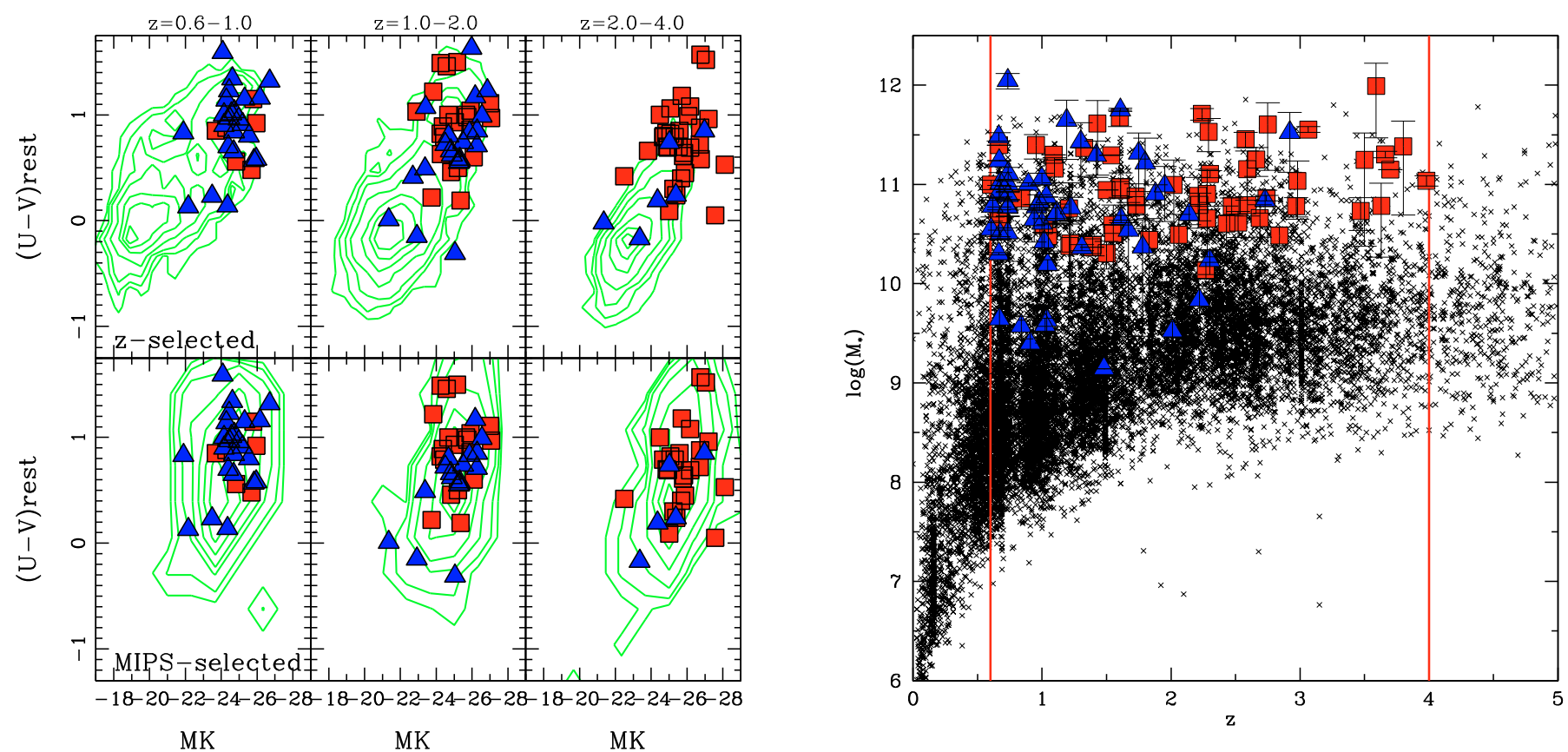

Fig. 8. Left panel: $U-V$ (rest frame) vs. the absolute $K$-band magnitude for the obscured AGN (squares) and the underlying galaxy population (contours). The upper panels show the comparison of the X-ray selected sources with the optically-selected (z-band) galaxy population in three different redshift bins: $z=0.6-1.0, z=1-2$, and $z=2.4$ from left to right, respectively. The lower panels show the comparison for the subsample of objects detected also at 24 micron, in the same redshift bins. The shapes and colors of the symbols reflect the AGN X-ray luminosity (see Fig. 5). Right panel: stellar mass $M_{*}$ vs. redshift for field galaxies (crosses) and our "obscured" AGN (squares and circles, same notation as in Fig. 5). The vertical lines mark the redshift range we chose for our analysis.
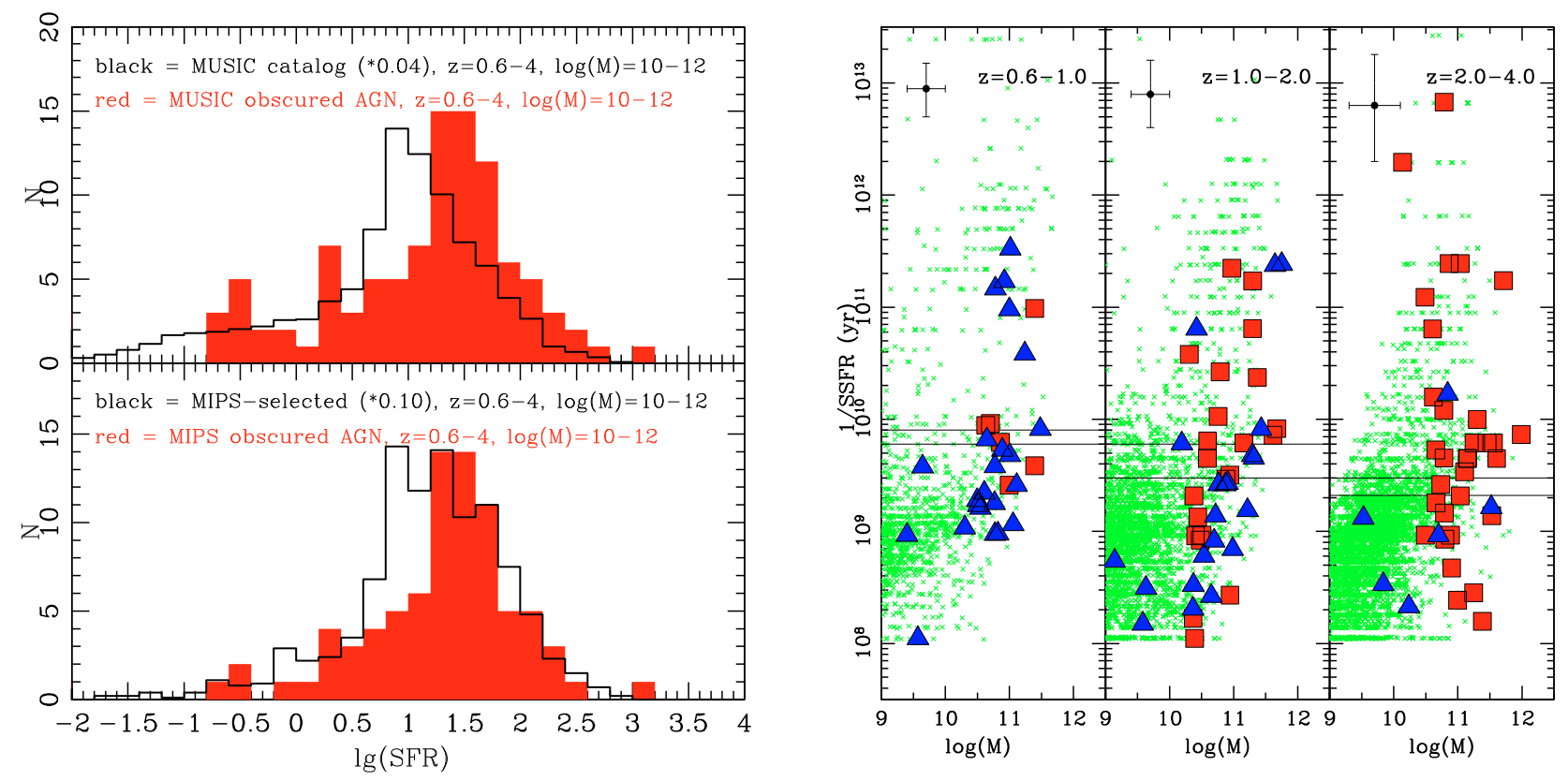

Fig. 9. (Left panel): the distribution of the SFR for both the optically (upper) and MIPS (lower) selected sample of field galaxies (black open histogram) and obscured AGN (red filled histogram) in the redshift interval $z=1-4$ and in a specific stellar mass range $\left(M_{*}=10^{10}-10^{12} M_{\odot}\right)$. (Right panel): inverse of the SSFR rate (defined as the inverse of the SFR per unit mass, $M / S F R$ ) as a function of the stellar mass of the galaxies in three different redshift bins for the overall field population (green crosses) and for the obscured AGN sample (filled symbols, color-coded on the basis of the AGN X-ray luminosity, see Fig. 5). In each panel the representative statistical error is also plotted as reference. The horizontal lines mark the age of the Universe at the two redshift boundaries of the chosen intervals.

In $\sim 65 \%$ of the cases the host galaxies of these AGN show substantial $\left(>10 M_{\odot} \mathrm{yr}^{-1}\right)$ amount of SFR. The distribution of SFR is significantly different from the one of optically selected galaxies (the probability that the two distributions shown in the upper-left panel of Fig. 9 are drawn from the same parent population is only $0.1 \%$, according to a Kolmogorov-Smirnov statistics) suggesting an enhancement of star formation activity in objects hosting obscured AGN. This result is solid despite the large 
systematics uncertainties (dominating the SFR measurements, see discussion in Sect. 2.4), given that they are expected to be present in both the optically and X-ray selected population.

An enhancement of the SFR activity in obscured AGN is qualitatively in agreement with the results at $z \sim 0.7$ obtained from an X-ray selected sample of AGN from the XMM-COSMOS survey (Silverman et al. 2009). As already pointed out in Fig. 8, the distribution of the SFR in the AGN sample mirrors the one of the MIPS selected sample (Fig. 9, lower-left panel) suggesting that AGN activity and star formation are indeed tightly related phenomena.

For each AGN host galaxy we could then calculate the Specific Star-Formation Rate (SSFR), defined as the instantaneous ratio of SFR to the total stellar mass $\left(S S F R=S F R / M_{*}\right)$, and its inverse, $1 / S S F R$, the so called "growth time", i.e. the time it would take a galaxy to double its stellar mass if forming stars at the observed instantaneous rate. Objects with growth time lower than the age of the Universe at their redshift $\left(1 / S S F R<t_{\text {Hubble }}\right)$ are actively forming stars (see, e.g. Fontana et al. 2009), while objects with growth time larger than the age of the Universe $\left(1 / S S F R>t_{\text {Hubble }}\right)$ can be considered dominated by a quiescent population. We will name hereafter "active host galaxies" the former, and "inactive host galaxies" the latter, when referring to the AGN hosts.

The right panel of Fig. 9 shows the growth time as a function of the stellar mass of the galaxies in three different redshift bins for the overall field population (green crosses) and for the obscured AGN sample (filled symbols, color-coded on the basis of the X-ray luminosity, see Fig. 5). The horizontal lines mark the age of the Universe at the two redshift boundaries of the chosen intervals. Taking the estimates of the SSFR at their face values, the majority ( $\geq 50 \%$ ) of obscured AGN in each redshift interval is hosted in "active host galaxies" confirming the trend outlined in Fig. 9 (left panel). However, we should note that in the highest redshift bin, the SSFR estimates suffer also from large statistical uncertainties (see error bars in the upper-left part of each panel in Fig. 9, right), and, as a consequence, the distinction between "active" and "inactive" host galaxies is rather fuzzy. Therefore, the results for this bin should be taken only as indicative.

\subsection{AGN fraction}

In the following we derive the AGN fraction as a function of the stellar mass of the underlying galaxy population. The AGN fraction has been computed as the ratio of X-ray selected AGN over the overall field population in each mass bin. Given that at $z>2$ we can sample only objects with $L_{X}>10^{43} \mathrm{erg} \mathrm{s}^{-1}$, the fractions have been computed further imposing this threshold to the obscured AGN sample (i.e. considering only objects marked as squares in the figures of the paper) in all redshifts bins and, therefore, they should be considered representative of this AGN population only ${ }^{5}$.

Figure 10 shows the AGN fraction as a function of $M_{*}$ in three different redshift bins. We find that the fraction of objects hosting AGN increases with the stellar mass of the galaxy, being up to $30 \%$ for $M_{*} \geq 3 \times 10^{11} M_{\odot}$, while it is $<1 \%$ for $M_{*}=$ $10^{10}-3 \times 10^{10} M_{\odot}$. We should note that the MUSIC sample cannot be considered complete for galaxies with masses $\geq 10^{10} M_{\odot}$ already at $z \sim 1$. Indeed, different levels of completeness affect

5 This threshold further assures that the observed X-ray emission is mostly due to AGN activity, with a negligible contribution from star-formation, even for the objects with the highest observed SFR (>1000 $M_{\odot} \mathrm{yr}^{-1}$, Ranalli et al. 2003).

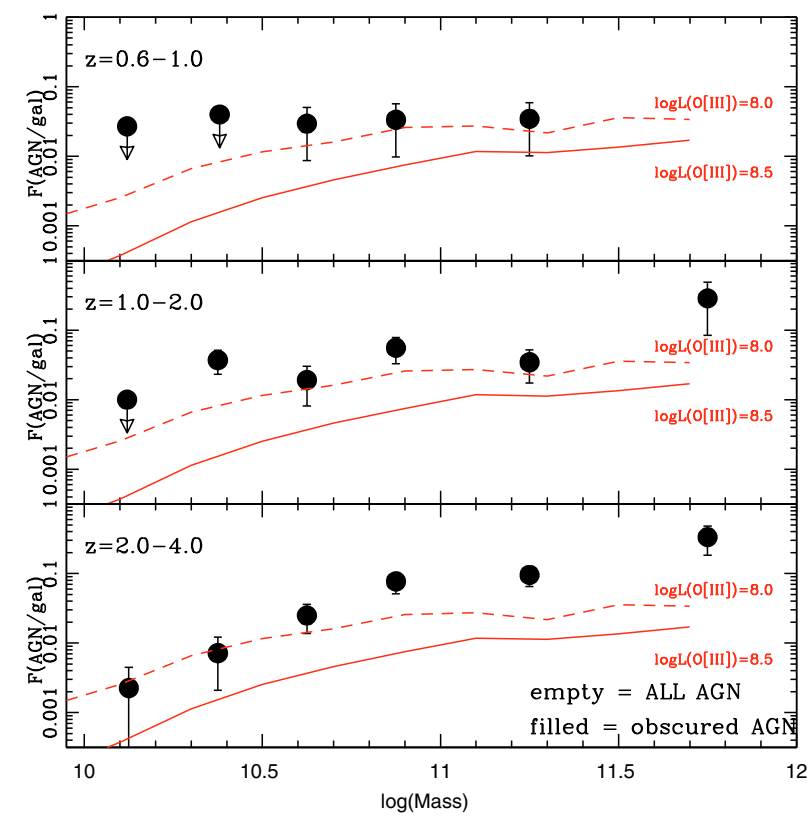

Fig. 10. Fraction of obscured AGN with $L_{\mathrm{X}}>10^{43} \mathrm{erg} \mathrm{s}^{-1}$ as a function of the stellar mass in three different redshift bins (see Fig. 8 for the definition). Filled circles refer to the "obscured" AGN sample. The dashed (continuous) lines represent the fraction of AGN with $L(\mathrm{OIII})>$ $10^{8.0}\left(10^{8.5}\right) L_{\odot}$ in an optically selected sample in the local Universe $(z<0.2)$ from the SDSS (Best et al. 2005). See text for details.

different kind of galaxies. The total sample is expected to be complete against passive galaxies with $M_{*} \geq 10^{11} M_{\odot}$ up to $z=4$. In particular, the stellar mass limit of star-forming galaxies, which have $M / L$ lower than passive galaxies, is lower than that of passive galaxies at all redshifts (Fontana et al. 2006). The incompleteness of the MUSIC sample for $M_{*} \geq 10^{11} M_{\odot}$ galaxies implies that the plotted AGN fractions are somewhat higher than the real fractions at those masses, and that the actual trends of increasing AGN fraction as a function of the stellar mass is probably steeper than those in Fig. 10.

The observed trend of the AGN fraction increasing with the stellar mass means that, for a given mass-selected sample, AGN will populate the high-mass tail of the parent sample mass distribution. Qualitatively similar results are obtained by Bundy et al. (2008) and Yamada et al. (2009). This therefore also explains the position of the AGN in the left panel of Fig. 8, i.e. among the most luminous, massive and reddest sources. Best et al. (2005) found a similar behaviour for the AGN fraction as a function of the stellar mass in SDSS AGN samples, selected on the basis of line ratios diagnostic diagrams (Baldwin et al. 1981; Kauffmann et al. 2003). In Fig. 10 we also plot the results from Best et al. (2005) corresponding to AGN with $L([\mathrm{OIII}])$ luminosity larger than $10^{8.0}$ and $10^{8.5} L_{\odot}$, as labeled. Using the relation between the observed $L(\mathrm{OIII})$ and the X-ray luminosity given by Netzer et al. (2006, Eq. (1)), these $L([\mathrm{OIII}])$ luminosities represent a fiducial range for local objects with $\mathrm{X}$-ray luminosities similar to those in our sample $\left(L_{\mathrm{X}}>10^{43} \mathrm{erg} \mathrm{s}^{-1}\right)$ when an average extinction $E(B-V)=0.4 \pm 0.2$ is taken into account (see discussion in Netzer et al. 2006).

It is interesting to note, however, that the increase with mass of the fraction of radio selected AGN (i.e. objects in which the radio emission is not related to star formation processes) is much steeper than what we find for our sample of AGN. This is true both locally (Best et al. 2005) and up to $z \sim 1.3$ (Smolcic et al. 2009). This difference in the mass-dependence of the fraction of 
radio versus X-ray or optically selected AGN is likely to be due to different accretion modes in these different classes of AGN (Smolcic et al. 2009).

\subsection{Eddington ratio distribution function}

From our multiwavelength database we were able to estimate another important parameter for the characterization of AGN emission, namely its Eddington ratio $\left(L / L_{\text {Edd }}\right)$, which gives the ratio at which the AGN is emitting compared to its expected Eddington luminosity ( $\left.L_{\mathrm{Edd}}\right)$. Indeed, the ratio between the X-ray luminosity and the host galaxy stellar mass is proportional to the AGN Eddington ratio, the proportionality factor depending on the bolometric correction between the X-ray and the bolometric luminosity, and on the $M_{\mathrm{BH}} / M_{*}$ ratio. We derived the Eddington ratio distribution function for our sources as explained below.

First, we calculated the ratio between the X-ray luminosity and the stellar mass for each obscured AGN with $M_{*}>10^{10} M_{\odot}$ in our sample $\left(L_{X} / M_{*}\right)$. Figure 11 shows the distribution of this quantity for the sources in two different redshifts bins at $z>1$ (solid histograms in the upper left and bottom panels of Fig. 11). The median (seminterquartiles) values of the distributions of $\log L_{\mathrm{X}} / M_{*}$ are similar, $\log L_{\mathrm{X}} / M_{*}=32.21(0.61)$ and $\log L_{X} / M_{*}=32.73(0.42)$ for the two redshift bins, respectively. When only the sources with $L_{\mathrm{X}}>10^{43} \mathrm{erg} \mathrm{s}^{-1}$ are considered, the median values of the $\log L_{\mathrm{X}} / M_{*}$ distribution are almost identical, $\log L_{\mathrm{X}} / M_{*}=32.87$ and $\log L_{\mathrm{X}} / M_{*}=32.83$, respectively (dashed histograms).

In the $z=1-2$ redshift bin, where the uncertainties on the SSFR estimates are reasonably small and therefore we are able to divide the sample in "active" and "inactive" galaxies (see Sect. 4.2), we also plotted the distribution of $L_{\mathrm{X}} / M_{*}$ for the most luminous sources $\left(L_{\mathrm{X}}>10^{43} \mathrm{erg} \mathrm{s}^{-1}\right)$ separately for these two populations (upper right panel of Fig. 11). We found that AGN in "inactive" host galaxies tend to have lower $\log \left(L_{\mathrm{X}} / M_{*}\right)$ values (dashed histogram in the upper right panel of Fig. 11) than those in "active" host galaxies although the statistical significance of this result is only marginal, the probability that the two distributions are drawn from the same parent population being $5 \%$, according to a Kolmogorov-Smirnov statistics.

Then, we assumed the local relation between $M_{\mathrm{BH}}$ and $M_{*}$ (e.g. Marconi \& Hunt 2003, $M_{\mathrm{BH}} \sim 10^{-3} \times M_{*}$ ) to convert the stellar mass in a $\mathrm{BH}$ mass and calculate $L_{\mathrm{Edd}}$, and we applied a bolometric correction of 20 (e.g. Marconi et al. 2004) to derive the bolometric luminosity from the X-ray luminosity. With these assumptions, the median values of $L_{\mathrm{X}} / M_{*}$ correspond to $L / L_{\text {Edd }} \sim 0.02-0.08\left(L / L_{\text {Edd }} \sim 0.1\right.$ for the $\log L_{\mathrm{X}}>43$ subsample). We note that, given the systematics uncertainties affecting the derivation of the stellar masses (see discussion in 2.4), and the fact that total stellar masses are derived and not bulge masses, the derived BH massses are most likely upper limits, this translating in conservative (lower-limits) estimate of the $L / L_{\text {Edd }}$. On the other hand, recently, it has been suggested that the average $M_{\mathrm{BH}}$-host galaxy mass ratio at $z=1-2$ is higher by a factor $\sim 2$ than the local value (McLure et al. 2006; Peng et al. 2006; Merloni et al. 2009). Should this be the case, our estimates of $L / L_{\text {Edd }}$ should be reduced by a factor of $\sim 2$ at $z=1-2$ and possibly more in the higher redshift bin if the average $M_{\mathrm{BH}}-M_{*}$ ratio further increases to higher redshift.

\section{Discussions and conclusions}

We presented a new catalog of the counterparts of the 179 extragalactic X-ray sources detected in the $1 \mathrm{Ms}$ Chandra observation

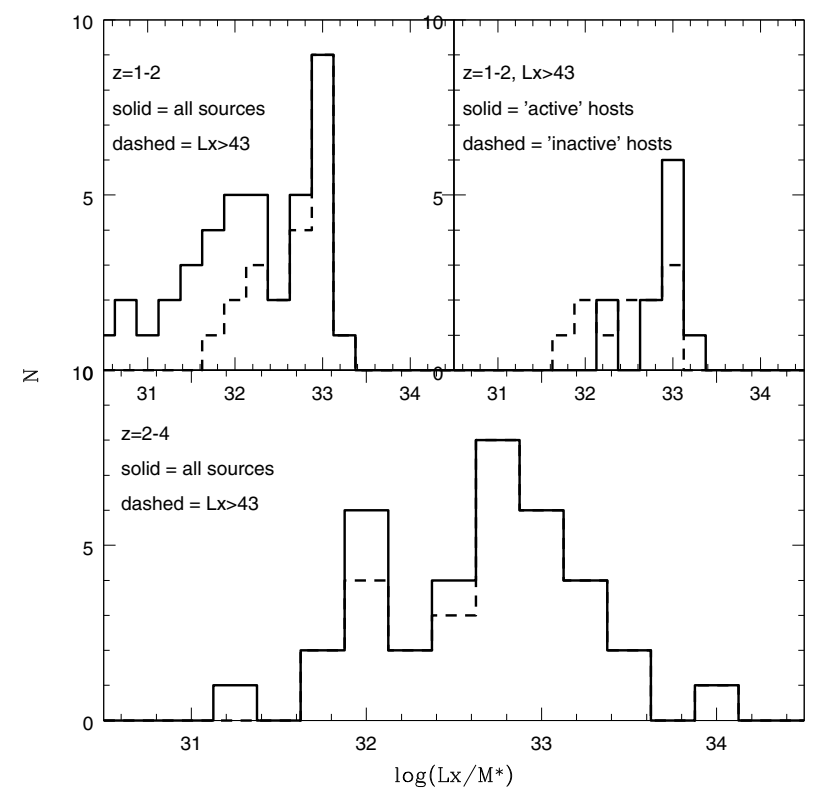

Fig. 11. The distribution of the ratio between the X-ray luminosity and the stellar mass for the obscured AGN in our sample (empty histograms), in two different redshifts bins as labeled.

of the MUSIC/CDFS/GOODS field and an extensive analysis of the host galaxies properties of obscured AGN.

We quantified the bias in the determination of the counterparts of X-ray selected sources when the match is limited to optical catalogs only, with respect to the combined use of optical and near infrared (deep $K$-band and IRAC) data. We estimate that the fraction of misidentified X-ray sources previously reported in the literature is of the order of $\sim 6 \%$, and rises up to $\sim 14 \%$ when optically faint $(z>24)$ sources are considered (see Fig. 1); the use of an optically-based catalog biases the identification against the most extreme, obscured sources therefore preventing the exact knowledge of the multiwavelength properties of the X-ray counterparts.

In order to study the host-galaxies of obscured AGN, we defined a sample of 116 "bona fide" obscured AGN, by selecting sources without broad lines in the optical spectra and with small optical nuclear emission with respect to the host galaxy optical emission (see Fig. 3). From eyeball inspection of the host galaxy morphology, we found a variety of cases, and a disturbed morphology (due to activity/merging/star formation) in more than half of the sub-sample for which a morphological classification could be made (see Fig. 4).

We investigated the optical to infrared colors of these obscured AGN. The most striking result is that half of the X-ray selected obscured AGN in the redshift range $z=1.5-4.0$ have a $8.0 \mu \mathrm{m}$ to $4.5 \mu \mathrm{m}$ flux ratio $<2$ and according to Pope et al. (2008) would have been classified as "star-formation" dominated objects (see Fig. 6); $50 \%$ of them in addition have $24.0 \mu \mathrm{m}$ to $8.0 \mu \mathrm{m}$ flux ratio $<5$, where the original Pope et al. (2008) diagram is almost empty. Moreover, previous analysis based on Chandra stacking analysis of sources with high MIR/O (Daddi et al. 2007a; Fiore et al. 2008, 2009) claimed a large contribution $(\geq 80 \%)$ of heavily obscured (Compton Thick) sources among the stacked population (but see also discussion in Donley et al. 2008). This suggests that 1 ) the accretion activity in highredshift sources is unambiguously revealed thanks to the presence of a strong X-ray emission; and 2) the star-formation region as defined by Pope et al. (2008) contains not only objects 
in which the bolometric luminosity is dominated by the starformation processes, but also a not-negligible number of objects hosting candidate obscured/Compton Thick AGN. In conclusion, our obscured AGN show Spitzer colors consistent with both an AGN dominated continuum and a starburst dominated continuum in the MIR.

We found that the hosts of obscured AGN are redder in $(U-V)$ rest frame than the overall galaxy population at the same redshift: in particular, obscured AGN mainly populate the red sequence and the green valley in the color-magnitude plots (Fig. 7, left panel), in agreement with the results of Nandra et al. (2007), and Silverman et al. (2007). For the MUSIC sample the $U-V$ galaxy colors are strongly correlated with the $K$ band absolute magnitude (Fig. 8, left panel), and therefore with the galaxy stellar mass, with the most massive systems having a redder color. The hosts of the obscured AGN are therefore found in the red-massive tail of the distribution of optically selected galaxies in all three redshift bins considered. AGN feedback is often invoked as one of the main responsible for the observed galaxy colors (Nandra et al. 2007; Hasinger 2008). However, it is well known that the main ingredient for nuclear activity is the presence of a SMBH in galaxy nuclei, and that SMBHs are found nearly exclusively in massive galaxies (e.g. Magorrian et al. 1998). Therefore, it is not truly surprising to find AGN hosted in massive galaxies and the simple presence of AGN in massive red galaxies is not enough to argue for a significant feedback effect on the observed colors, because of the strong color-mass correlation. Were AGN feedback responsible for the observed red colors, since galaxy colors are strongly correlated with the galaxy mass and AGN are found preferably in massive galaxies, then AGN feedback should be also considered as one of the main players in the building of the galaxy mass-color correlation.

We found that about $2 / 3$ of the obscured AGN hosts at all redshifts show substantial $\left(>10 M_{\odot} \mathrm{yr}^{-1}\right)$ star formation activity (Fig. 9 left panel) and about half live in galaxies which are still actively forming stars with respect to their mass (Fig. 9, right panel). For these sources, the observed red colors are likely due to dust extinction rather than evolved stellar population. We then conclude that a significant fraction of obscured AGN live in massive, dusty star-forming galaxies with red optical colors. This result is in qualitative agreement with the morphological analysis. Higher luminosity X-ray selected AGN are not systematically found in objects with the highest SSFR (see Fig. 9 right panel), in agreement with Alonso-Herrero et al. (2008).

We compared the number of obscured AGN and of all X-ray selected AGN to the number of field galaxies in broad bins of galaxy stellar mass $\left(M_{*}=10^{10}-10^{12} M_{\odot}\right)$ and redshifts $(z=0.6-1, z=1-2, z=2-4)$. We find that the AGN fraction increases with the host galaxy stellar mass, from $\sim 1 \%$ at $M_{*} \sim 10^{10} M_{\odot}$ to $\sim 30 \%$ at $M_{*} \sim 3 \times 10^{11} M_{\odot}$ (see also Yamada et al. 2009), and the actual trend of increasing AGN fraction as a function of the stellar mass is probably steeper given the uncompleteness of the MUSIC sample at $M_{*}<10^{11} M_{\odot}$ (see Sect. 4.4). The uncertainties on the stellar mass estimate from the SED fitting have the effect of shifting at lower masses (of $\sim 0.25 \mathrm{dex}$ ) the datapoints, leaving the total fraction and the slope unchanged.

We compared this trend with that observed in the local Universe (Best et al. 2005) for AGN with luminosity above similar thresholds. While the observed trend is the same, in all the investigated redshift bins the AGN fraction is higher than that observed in the local Universe, and it could likely be even higher. In fact, we are comparing here AGN selected with two different methods: forbidden emission line luminosity (SDSS) and X-ray emission (GOODS). The latter sample does not contain most Compton thick AGN. On the other hand, Compton thick AGN may well be present in [OIII] selected AGN samples. The fraction of Compton thick AGN not directly detected in deep Chandra surveys is estimated between $40 \%$ and $100 \%$ of the X-ray selected AGN, using infrared selection or other techniques (see Donley et al. 2008; Fiore et al. 2009, and references therein). Therefore, under the simplest assumption that this Compton Thick AGN fraction is constant with the galaxy mass, the discrepancy observed in Fig. 10 can increase by up of a factor of 2 .

The fraction of active galaxies to the total galaxy population is proportional to the AGN duty cycle. Our results would thus suggest higher AGN duty cycles at $z=2-4$ than at $z=0$, in agreement with expectations from most recent semi-analytic models (e.g. Menci et al. 2008), in which at higher redshift the AGN activity is present in a larger number of galaxies than locally.

The fact that the most luminous obscured AGN are found in the most massive galaxies at all investigated redshifts may suggest that the $L / L_{\text {Edd }}$ of the obscured AGN is similar, particularly in the case of the most luminous sources $\left(\log L_{X}>43 \mathrm{erg} \mathrm{s}^{-1}\right)$, for which the threshold in luminosity introduces a bias against the sources accreting at lower rates in the lowest redshift bin. Assuming the local Magorrian relation between $M_{\mathrm{BH}}$ and $M_{*}$ (e.g. Marconi \& Hunt 2003) and a bolometric correction of 20 (e.g. Marconi et al. 2004) the median values of $L_{X} / M_{*}=32.83$ and $L_{X} / M_{*}=32.87$ correspond to $L / L_{\text {Edd }} \sim 0.1$. Although suffering from large uncertainties associated with the stellar mass and $\mathrm{BH}$ mass estimates (see Sect. 4.4), this value can be considered as representative of the accretion state of the most luminous, obscured AGN in the present sample. Similar results are obtained from a comprehensive analysis of host galaxies properties of Chandra Deep Field North X-ray sources sources at $z=2-4$ (Yamada et al. 2009) and are also typical of unobscured type 1 AGN at $z>1$ (Merloni et al. 2009; Trump et al. 2009).

It is instructive to compare these findings with similar estimates in the local Universe. Kauffmann \& Heckman (2009) using SDSS data found an average $L / L_{\text {Edd }}$ value of $\sim 0.01$ and a log normal distribution for this parameter, for AGN hosted in galaxies with significant on-going star formation, while AGN in inactive host galaxies follow a power-law distribution. As discussed in Sect. 4.4, and shown in the upper right panel of Fig. 11, we also find marginal evidence of different accretion rates distributions for the populations of AGN in "active" and "inactive" host galaxies.

\section{Summary}

Taking advantage of the 1Ms CDFS Chandra observations and the associated deep multiwavelength follow-up, we have studied the host galaxies properties of obscured AGN at $z>1$.

The most important results of our analysis can be summarized as follows:

- X-ray selected $\left(L_{\mathrm{X}} \gtrsim 10^{42} \mathrm{erg} \mathrm{s}^{-1}\right)$ AGN show Spitzer colors consistent with both AGN and starburst dominated infrared continuum and only the combination of the two selections (X-ray + Mid-infrared) can fully characterize the growth of SMBH at $z>1$;

- the host galaxies of X-ray selected obscured AGN are all massive and in $50 \%$ of the cases are also actively forming stars. Their red colors are due to dust extinction; 
- the X-ray selected AGN fraction increases with the stellar mass up to a value of $\sim 30 \%$ at $z>1$ and $M_{*}>3 \times 10^{11} M_{\odot}$, a fraction significantly higher than what is observed in the local Universe $(\sim 2 \%)$ for AGN of similar luminosities;

- the median $L / L_{\text {Edd }}$ value is between $2 \%$ and $10 \%$ depending on the assumed $M_{\mathrm{BH}} / M_{*}$ ratio, the X-ray luminosity threshold and the host galaxies properties of the obscured AGNs.

Acknowledgements. The authors are grateful to P. N. Best for deriving the SDSS OIII source fractions as a function of stellar mass with an optical luminosity cut that matches the present X-ray data. M.B. and F.F. acknowledge useful discussions with Roberto Gilli, Paolo Tozzi, John Silverman, Cristian Vignali, G. Cesare Perola, Silvia Bonoli, Niel Brandt, Bin Luo, Hagai Netzer and Jochen Greiner. We thank the anonymous referee for insightful and detailed comments which helped in the presentation of the results. M.B. acknowledges support from the XMM-Newton DLP grant 50-)G-0502; F.F. and A.C. acknowledge support from ASI contracts I/088/06/0 and I/016/07/0 and PRIN/MIUR 2006-02-5203.

\section{References}

Alexander, D. M., Bauer, F. E., Brandt, W. N., et al. 2003, AJ, 126, 539 Alexander, D. M., Bauer, F. E., Chapman, S. C., et al. 2005, ApJ, 632, 736 Alonso-Herrero, A., Perez-Gonzalez, P. G., Rieke, G. H., et al. 2008, ApJ, 677, 127

Antonucci, R. R. 1993, ARA\&A, 31, 473

Antonucci, R. R., \& Miller, J. S. 1985, ApJ, 297, 621

Baldwin, J. A., Phillips, M. M., \& Terlevich, R. 1981, PASP, 93, 5

Bauer, F. E., Alexander, D. M., Brandt, W. N., et al. 2004, AJ, 128, 2048

Best, P. N., Kauffmann, G., Heckman, T. M., et al. 2005, MNRAS, 362, 35

Brusa, M., Comastri, A., Daddi, E., et al. 2005, A\&A, 432, 69

Brusa, M., Zamorani, G., Comastri, A., et al. 2007, ApJS, 172, 353

Bruzual, G., \& Charlot, S. 2003, MNRAS, 344, 1000

Bundy, K., Georgakakis, A., Nandra, K., et al. 2008, ApJ, 681, 931

Cardamone, C. N., Urry, C. M., Damen, M., et al. 2008, ApJ, 680, 130

Cristiani, S., Appenzeller, I., Arnouts, S., et al. 2000, A\&A, 359, 489

Comastri, A., \& Brusa, M. 2008, AN, 329, 122

Daddi, E., Renzini, A., Pirzkal, N., et al. 2005, ApJ, 626, 680

Daddi, E., Alexander, D. M., Dickinson, M., et al. 2007a, ApJ, 670, 173

Daddi, E., Dickinson, M., Morrison, G., et al. 2007b, ApJ, 670, 156

De Santis, C., Grazian, A., Fontana, A., \& Santini, P. 2007, New Astron., 12, 271

Dey, A., Soifer, B. T., Desai, V., et al. 2008, ApJ, 677, 943

Di Matteo, T., Springel, V., \& Hernquist, L. 2005, Nature, 433, 604

Donley, J. L., Rieke, G. H., Perez-Gonzalez, P. G., \& Barro, G. 2008, ApJ, 687, 111

Fabian, A. C. 1999, MNRAS, 308, L39

Ferreras, I., Lisker, T., Carollo, C. M., Lilly, S. J., \& Mobasher, B., 2005, ApJ, 635,243

Fiore, F., Grazian, A., Santini, P., et al. 2008, ApJ, 692, 74

Fiore, F., Puccetti, S., Brusa, M., et al. 2009, ApJ, 693, 447

Fontana, A., Salimbeni, S., Grazian, A., et al. 2006, 459, 745

Fontana, A., Santini, P., Grazian, A., et al. 2009, A\&A, 501, 15

Georgantopoulos, I., Georgakakis, A., \& Akylas, A. 2007, A\&A, 466, 823

Georgantopoulos, I., Georgakakis, A., Rowan-Robinson, M., \& Rovilos, E. 2008, A\&A, 484, 671

Giacconi, R., Zirm, A., Wang, J., et al. 2002, ApJS, 139, 369

Giavalisco, M., Ferguson, H. C., Koekemoer, A. M., et al. 2004, ApJ, 600, L93

Gilli, R., Daddi, E., Zamorani, G., et al. 2005, A\&A, 430, 811

Gilli, R., Comastri, A., \& Hasinger, G. 2007, A\&A, 463, 79

Granato, G. L., De Zotti, G., Silva, L., Bressan, A., \& Danese, L. 2004, ApJ, 600,580
Grazian, A., Fontana, A., de Santis, C., et al. 2006, A\&A, 449, 951 Hasinger, G. 2008, A\&A, 490, 905

Hickox, R., Jones, C., Forman, W. R., et al. 2009, ApJ, 696, 891

Hopkins, P. F., Hernquist, L., Cox, T. J., \& Keres, D. 2008, ApJS, 175, 365

Kauffmann, G., \& Heckman, T. M. 2009, MNRAS, 397, 153

Kauffmann, G., Heckman, T. M., Tremonti, C., et al. 2003, MNRAS, 346, 1055 Koekemoer, A. M., Alexander, D. M., Bauer, F. B., et al. 2004, ApJ, 600, L123 Ilbert, O., capak, P., Salvato, M., et al. 2009, ApJ, 690, 1236

Ivison, R. J., Greve, T. R., Serjeant, S., et al. 2004, ApJS, 154, 124

Le Fevre, O., Vettolani, G., Paltani, S., et al. 2004, A\&A, 428, 1043

Lehmer, B., Brandt, W. N., Alexander, D. M., et al. 2005, ApJS, 161, 21

Magorrian, J., Tremaine, S., Richstone, D., et al. 1998, AJ, 115, 2285

Mainieri, V., Rosati, P., Tozzi, P., et al. 2005, A\&A, 437, 805

Marconi, A., \& Hunt, L. K. 2003, ApJ, 589, L21

Marconi, A., Risaliti, G., Gilli, R., et al. 2004, MNRAS, 351, 169

Martinez-Sansigre, A., Rawlings, S., Lacy, M., et al. 2005, Nature, 436, 666

Martinez-Sansigre, A., Rawlings, S., Lacy, M., et al. 2006, MNRAS, 370, 1479

McLure, R. J., Jarvis, M. J., Targett, T. A., Dunlop, J. S., \& Best, P. N. 2006, MNRAS, 368, 1395

Menci, N., Fontana, A., Giallongo, E., Grazian, A., \& Salimbeni, S. 2006, ApJ, 647,753

Menci, N., Fiore, F., Puccetti, S., \& Cavaliere, A. 2008, ApJ, 686, 219

Merloni, A., \& Heinz, S. 2008, MNRAS, 338, 1011

Merloni, A., Bongiorno, A., Bolzonella, M., et al. 2009, ApJ, in press, [arXiv: 0910.4970]

Mignoli, M., Cimatti, A., Zamorani, G., et al. 2005, A\&A, 437, 883

Netzer, H., Mainieri, V., Rosati, P., \& Trakhtenbrot, B. 2006, A\&A, 453, 525

Nandra, K., Georgakakis, A., Willmer, C. N. A., et al. 2007, ApJ, 660, L11

Norman, C., Hasinger, G., Giacconi, R., et al. 2002, ApJ, 571, 218

Page, M. J., Stevens, J. A., Ivison, R. J., \& Carrera, F. J. 2004, ApJ, 611, L85

Papovich, C., Rudnick, G., Le Floch, E., et al. 2007, ApJ, 668, 45

Peng, C. Y., Impey, C. D., Rix, H.-W., et al. 2006, ApJ, 649, 616

Polletta, M., Tajer, M., Maraschi, L., et al. 2007, ApJ, 663, 81

Pope, A., Bussman, R. S., Dey, A., et al. 2008, ApJ, 689, 127

Popesso, P., Dickinson, M., Nonino, M., et al. 2009, A\&A, 494, 443

Pozzi, F., Vignali, C., Comastri, A., et al. 2007, A\&A, 468, 603

Ranalli, P., Comastri, A., \& Setti, G. 2003, A\&A, 399, 39

Ranalli, P., Comastri, A., \& Setti, G. 2005, A\&A, 440, 23

Rigby, J. R., Rieke, G. H., Perez-Gonzalez, P. G., et al. 2005, ApJ, 627, 134

Rodighiero, G., Cimatti, A., Franceschini, A., et al. 2007, A\&A, 470, 21

Rovilos, E., \& Georgantopoulos, I. 2007, A\&A, 475, 115

Salpeter, E. E. 1955, ApJ, 121, 161

Santini, P., Fontana, A., Grazian, A., et al. 2009, A\&A, 504, 751

Silk, J., \& Rees, M. J. 1998, A\&A, 311, L1

Silverman, J. D., Green, P. J., Barkhouse, W. A., et al. 2008, ApJ, 679, 118

Silverman, J. D., et al. 2009, ApJ, in press

Smolcic, V., Zamorani, G., Schinnerer, E., et al. 2009, ApJ, 696, 24

Stern, D., Eisenhardt, P., Gorjian, V., et al. 2005, ApJ, 631, 163

Stevens, J. A., et al. 2006, MNRAS, 360, 610

Szokoly, G. P., Bergeron, J., Hasinger, G., et al. 2004, ApJS, 155, 271

Tozzi, P., Rosati, P., Nonino, M., et al. 2001, ApJ, 562, 42

Tozzi, P., Gilli, R., Mainieri, V., et al. 2006, A\&A, 451, 457

Tozzi, P., Mainieri, V., Rosati, P., et al. 2009, ApJ, 698, 740

Trump, J. R., Impey, C. D., Kelly, B. C., et al. 2009, ApJ, 700, 49

Urry, C. M., \& Padovani, P. 1995, PASP, 107, 803

Vanzella, E., Cristiani, S., Dickinson, M., et al. 2005, A\&A, 434, 53

Vanzella, E., Cristiani, S., Dickinson, M., et al. 2006, A\&A, 454, 23

Vanzella, E., Cristiani, S., Dickinson, M., et al. 2008, A\&A, 478, 83

Wolf, C., Meisenheimer, K., Kleinheinrich, M., et al. 2004, A\&A, 421, 913

Yamada, T., Kajisawa, M., Akyiama, M., et al. 2009, ApJ, 699, 1354

Yan, L., Sajina, A., Fadda, D., et al. 2007, ApJ, 658, 778

Zheng, W., Mikles, V. J., Mainieri, V., et al. 2004, ApJS, 155, 73 
M. Brusa et al.: Black hole growth and starburst activity at $z=0.6-4$ in the Chandra Deep Field South, Online Material $p 1$

Table 1. Counterparts to CDFS X-ray sources: positions, fluxes, redshifts and luminosities.

\begin{tabular}{|c|c|c|c|c|c|c|c|c|c|c|}
\hline 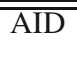 & "XID & 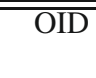 & $\begin{array}{c}\text { RA } \\
\text { J2000 }\end{array}$ & $\begin{array}{c}\text { Dec } \\
\text { J2000 }\end{array}$ & $\begin{array}{r}\text { dist } \\
\operatorname{arcsec}\end{array}$ & $\overline{\text { FLAG }}$ & $\begin{array}{c}S_{2-10} \mathrm{keV} \\
10^{-15} \mathrm{cgs}\end{array}$ & $\begin{array}{c}S_{0.5-2} \mathrm{keV} \\
10^{-15} \mathrm{cgs}\end{array}$ & $\overline{\overline{\text { Redshift }}}$ & $\begin{array}{l}\overline{l o g}\left(L_{X}\right) \\
\text { erg s }^{-1}\end{array}$ \\
\hline 51 & $108 \mathrm{a}$ & 13272 & 53.024029 & -27.746366 & 0.32 & $0^{m}$ & 1.13 & 0.41 & $1.608(3)$ & 43.33 \\
\hline 53 & $560^{*}$ & 12222 & 53.026127 & -27.760183 & 0.22 & 0 & $<0.51$ & 0.15 & $0.669(1)$ & 41.63 \\
\hline 67 & 221 & 70103 & 53.036953 & -27.740129 & 0.34 & $0^{k, m}$ & $<0.52$ & 0.15 & $2.43(0.7-4.5)$ & 43.13 \\
\hline 71 & 61 & 70051 & 53.043751 & -27.719135 & 0.12 & 0 & 12.28 & 5.68 & $3.59(3.4-4.3)$ & 45.04 \\
\hline 73 & 536 & 15717 & 53.044842 & -27.709606 & 0.34 & 0 & $<0.87$ & 0.11 & $0.418(4)$ & 41.26 \\
\hline 75 & 185 & 14349 & 53.045467 & -27.728628 & 1.66 & 0 & $<0.61$ & 0.09 & 0.998 (3) & 42.55 \\
\hline 76 & 60 & 13849 & 53.045471 & -27.737484 & 0.16 & 0 & 11.89 & 6.66 & $1.615(1)$ & 44.23 \\
\hline 77 & 80 & 8168 & 53.045666 & -27.815575 & 0.03 & 0 & 1.64 & 0.93 & $1.39(1.2-3.0)$ & 43.21 \\
\hline 80 & 535 & 10619 & 53.047535 & -27.780546 & 0.21 & 0 & 1.28 & 0.26 & $0.575(1)$ & 42.23 \\
\hline 82 & 58 & 11180 & 53.049049 & -27.774496 & 0.02 & $0^{m}$ & 2.18 & 0.79 & $2.21(1.6-4.0)$ & 43.81 \\
\hline 83 & 534 & 12281 & 53.050819 & -27.758352 & 0.73 & 0 & $<0.55$ & 0.07 & $0.676(2)$ & 42.27 \\
\hline 84 & 149 & 11285 & 53.050934 & -27.772402 & 0.42 & 0 & 1.19 & 0.17 & $1.033(2)$ & 42.79 \\
\hline 88 & $56 \mathrm{a}$ & 15626 & 53.055141 & -27.711374 & 0.09 & 0 & 17.50 & 2.81 & $0.605(1)$ & 43.4 \\
\hline 90 & 600 & 12434 & 53.057587 & -27.757130 & 0.14 & 0 & 0.66 & $<0.06$ & $1.327(2)$ & 43.13 \\
\hline 91 & 266 & 15382 & 53.057747 & -27.713591 & 0.73 & 0 & 1.01 & $<0.08$ & $0.735(1)$ & 42.81 \\
\hline 93 & 583 & 70323 & 53.057854 & -27.833355 & 0.78 & $0^{k, m}$ & $<0.48$ & 0.19 & $2.24(1.2-2.3)$ & 43.6 \\
\hline 94 & 55 & 5434 & 53.058353 & -27.850199 & 0.12 & 0 & 7.78 & 0.83 & $0.122(1)$ & 41.51 \\
\hline 95 & 532 & 16040 & 53.058666 & -27.708439 & 0.17 & 0 & $<0.85$ & 0.16 & $2.026(4)$ & 42.83 \\
\hline 96 & 531 & 5281 & 53.060116 & -27.853006 & 0.18 & 0 & 0.97 & 0.07 & $1.544(1)$ & 43.46 \\
\hline 99 & 593 & 14104 & 53.061623 & -27.734022 & 1.84 & $0^{m}$ & $<0.59$ & $<0.10$ & $1.67(1.3-2.25)$ & 42.44 \\
\hline 100 & $82 \mathrm{a}$ & 5440 & 53.061905 & -27.851044 & 0.17 & $0^{m}$ & 1.46 & 0.27 & $1.21(0.8-2.6)$ & 43.05 \\
\hline 102 & 83 & 16094 & 53.062420 & -27.706909 & 0.29 & 0 & 3.29 & 1.04 & $2.494(4)$ & 43.56 \\
\hline 103 & 53 & 4878 & 53.062420 & -27.857515 & 0.18 & 0 & 4.00 & 1.93 & $0.675(1)$ & 42.86 \\
\hline 106 & 552 & 2654 & 53.065826 & -27.890200 & 0.35 & 0 & $<0.40$ & 0.28 & $0.674(1)$ & 41.91 \\
\hline 111 & $218 b$ & 4384 & 53.068401 & -27.866474 & 0.33 & 0 & $<0.56$ & 0.29 & $1.48(1.7-2.6)$ & 42.72 \\
\hline 112 & 564 & 10944 & 53.069603 & -27.777264 & 0.09 & $0^{m}$ & 0.31 & 0.12 & $2.61(0.5-3.4)$ & 43.14 \\
\hline 113 & 173 & 14774 & 53.069737 & -27.724312 & 0.37 & 0 & $<0.58$ & 0.10 & $0.524(1)$ & 41.56 \\
\hline 114 & $635 \mathrm{a}$ & 6606 & 53.069878 & -27.835489 & 1.73 & 0 & $<0.32$ & 0.04 & $0.729(1)$ & 41.49 \\
\hline 115 & 575 & 7393 & 53.071068 & -27.822748 & 0.43 & 0 & 0.47 & 0.06 & $0.340(1)$ & 41.26 \\
\hline 116 & $205 \mathrm{a}$ & 17038 & 53.071293 & -27.693579 & 0.29 & 0 & 1.29 & 0.17 & $2.22(1.5-2.7)$ & 43.62 \\
\hline 117 & 52 & 15207 & 53.071426 & -27.717581 & 0.02 & 0 & 7.89 & 4.85 & $0.569(1)$ & 42.98 \\
\hline 118 & 51 & 3920 & 53.071545 & -27.872448 & 0.13 & 0 & 14.16 & 0.62 & 1.097 (1) & 44.02 \\
\hline 121 & 566 & 9792 & 53.075058 & -27.788486 & 0.83 & 0 & $<0.41$ & 0.30 & $0.734(2)$ & 42.02 \\
\hline 122 & 87 & 3533 & 53.076000 & -27.878160 & 0.12 & 0 & 0.76 & 0.39 & $2.801(1)$ & 43.58 \\
\hline 123 & 153 & 5551 & 53.076405 & -27.848669 & 0.05 & 0 & 5.06 & 0.19 & $1.536(1)$ & 44.17 \\
\hline 124 & $526 \mathrm{a}$ & 13866 & 53.077923 & -27.736874 & 1.06 & 0 & 0.66 & 0.09 & $0.958(1)$ & 42.46 \\
\hline 125 & $263 b$ & 4835 & 53.078468 & -27.859856 & 0.73 & $0^{m}$ & 1.11 & 0.08 & $3.660(1)$ & 44.13 \\
\hline 126 & 50 & 9279 & 53.079144 & -27.798740 & 0.10 & 0 & 2.21 & 0.37 & $0.670(1)$ & 42.61 \\
\hline 129 & 525 & 17067 & 53.082535 & -27.689650 & 0.36 & 0 & $<0.97$ & 0.25 & $0.229(1)$ & 40.78 \\
\hline 130 & 524 & 70052 & 53.083061 & -27.711782 & 0.85 & $0^{m}$ & 1.23 & 0.22 & $1.50(0.0-5.5)$ & 43.19 \\
\hline 131 & 253 & 13250 & 53.083542 & -27.746447 & 0.32 & 0 & 2.99 & 0.13 & $0.481(1)$ & 42.47 \\
\hline 133 & 523 & 70044 & 53.084816 & -27.707909 & 0.07 & $0^{k, m}$ & 0.65 & 0.13 & $>3.50$ & $>43.76$ \\
\hline 134 & 151 & 9723 & 53.085323 & -27.792313 & 0.14 & 0 & 4.34 & 0.07 & $0.604(2)$ & 42.98 \\
\hline 136 & $522 \mathrm{a}^{*}$ & 489 & 53.089176 & -27.930479 & 0.43 & 0 & $<0.97$ & 0.47 & $2.611(4)$ & 43.73 \\
\hline 137 & $612 b$ & 15948 & 53.089264 & -27.708660 & 0.87 & 0 & 1.24 & $<0.08$ & $0.736(1)$ & 42.8 \\
\hline 139 & 602 & 10384 & 53.091606 & -27.782188 & 0.60 & 0 & 0.38 & $<0.06$ & $0.668(1)$ & 42.34 \\
\hline 141 & 572 & 70249 & 53.092484 & -27.803045 & 0.71 & $0^{m}$ & $<0.50$ & 0.07 & $2.75(1.5-3.8)$ & 43.37 \\
\hline 143 & $561 \mathrm{a}$ & 12146 & 53.093548 & -27.762192 & 0.13 & $0^{m}$ & $<0.36$ & 0.10 & $2.14(1.1-3.8)$ & 42.64 \\
\hline 145 & 145 & 11723 & 53.093922 & -27.767733 & 0.07 & 0 & 3.84 & 0.38 & $1.727(6)$ & 43.87 \\
\hline 146 & 188 & 7031 & 53.093948 & -27.830509 & 0.16 & 0 & 0.59 & 0.10 & $0.734(1)$ & 42.15 \\
\hline 149 & 521 & 3781 & 53.094841 & -27.873321 & 0.50 & 0 & 0.81 & 0.26 & $0.131(1)$ & 40.56 \\
\hline 151 & 204 & 11854 & 53.096493 & -27.765190 & 0.26 & 0 & 0.40 & 0.07 & $1.223(2)$ & 42.51 \\
\hline 152 & 627 & 8029 & 53.097259 & -27.814608 & 0.52 & 0 & $<0.30$ & 0.07 & $0.248(1)$ & 40.94 \\
\hline 154 & 241 & 15419 & 53.100811 & -27.715981 & 0.10 & 0 & $<0.65$ & 0.26 & $2.303(4)$ & 43.12 \\
\hline 155 & 49 & 17077 & 53.101070 & -27.690670 & 0.35 & 0 & 3.76 & 2.01 & $0.534(1)$ & 42.59 \\
\hline 157 & 598 & 1780 & 53.102806 & -27.903250 & 0.10 & 0 & $<0.62$ & $<0.09$ & 0.617 (1) & 42.72 \\
\hline 158 & 565 & 10250 & 53.103580 & -27.785107 & 0.35 & 0 & $<0.38$ & 0.10 & $0.363(2)$ & 40.93 \\
\hline 159 & 48 & 371 & 53.103531 & -27.933342 & 0.13 & 0 & 4.83 & 1.03 & 1.049 (3) & 43.4 \\
\hline 160 & $606 \mathrm{a}$ & 6640 & 53.103993 & -27.835571 & 0.19 & $0^{m}$ & 1.19 & 0.07 & $1.037(1)$ & 42.88 \\
\hline 162 & 260 & 5832 & 53.104610 & -27.845348 & 0.24 & 0 & 1.09 & 0.09 & $1.043(1)$ & 42.93 \\
\hline 163 & 46 & 16224 & 53.104858 & -27.705219 & 0.05 & 0 & 4.24 & 2.99 & $1.617(1)$ & 43.78 \\
\hline 164 & 150 & 1192 & 53.104832 & -27.913927 & 0.55 & 0 & 2.62 & $<0.09$ & $1.090(1)$ & 43.33 \\
\hline 166 & 45 & 15260 & 53.107006 & -27.718241 & 0.14 & $0^{m}$ & 5.04 & 1.22 & $2.291(1)$ & 44.22 \\
\hline 167 & 233 & 7194 & 53.107262 & -27.826765 & 0.25 & 0 & $<0.66$ & 0.09 & $0.577(1)$ & 42.14 \\
\hline
\end{tabular}


M. Brusa et al.: Black hole growth and starburst activity at $z=0.6-4$ in the Chandra Deep Field South, Online Material $p 2$

Table 1. continued.

\begin{tabular}{|c|c|c|c|c|c|c|c|c|c|c|}
\hline "AID & XID & $\overline{\mathrm{O} \text { OID }}$ & $\begin{array}{c}\text { RA } \\
\text { J2000 }\end{array}$ & $\begin{array}{c}\text { Dec } \\
\text { J2000 }\end{array}$ & $\begin{array}{r}\text { dist } \\
\operatorname{arcsec}\end{array}$ & 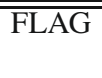 & $\begin{array}{c}S_{2-10} \mathrm{keV} \\
10^{-15} \mathrm{cgs}\end{array}$ & $\begin{array}{c}S_{0.5-2} \mathrm{keV} \\
10^{-15} \mathrm{cgs}\end{array}$ & Redshift & $\begin{array}{l}\log \left(L_{X}\right) \\
e^{-1 g ~ s}{ }^{-1}\end{array}$ \\
\hline 170 & 589 & 70385 & 53.107422 & -27.855698 & 0.66 & $0^{k, m}$ & $<0.38$ & 0.10 & $3.98(1.25-4.8)$ & 43.24 \\
\hline 171 & 519 & 978 & 53.107746 & -27.918447 & 1.09 & 0 & $<0.80$ & 0.20 & $1.034(1)$ & 42.41 \\
\hline 172 & 81 & 12747 & 53.108109 & -27.753985 & 0.21 & $0^{m}$ & 1.12 & 0.52 & $2.98(2.0-3.7)$ & 43.82 \\
\hline 175 & 518 & 70168 & 53.111542 & -27.767839 & 0.26 & $0^{m}$ & 0.63 & 0.10 & $>0.40$ & $>41.55$ \\
\hline 176 & 43 & 16792 & 53.111519 & -27.695988 & 0.06 & 0 & 3.57 & 0.69 & $0.734(1)$ & 42.91 \\
\hline 177 & $42 a$ & 17452 & 53.112530 & -27.684723 & 0.15 & 0 & 84.95 & 53.27 & 0.734 (1) & 44.27 \\
\hline 179 & 41 & 16831 & 53.115097 & -27.695806 & 0.19 & 0 & 8.81 & 0.55 & $0.668(1)$ & 43.24 \\
\hline 180 & $623 a$ & 10464 & 53.118420 & -27.783386 & 0.70 & 0 & $<0.20$ & 0.08 & $2.14(1.8-2.6)$ & 42.62 \\
\hline 181 & 224 & 11248 & 53.119724 & -27.772304 & 0.10 & 0 & 0.27 & 0.26 & $0.735(2)$ & 41.77 \\
\hline 182 & 103 & 13977 & 53.120071 & -27.732124 & 0.25 & 0 & 1.07 & 0.72 & $0.215(1)$ & 41.15 \\
\hline 187 & 599 & 2637 & 53.124100 & -27.891216 & 0.76 & $0^{m}$ & 0.67 & $<0.08$ & $2.59(2.0-4.0)$ & 43.62 \\
\hline 188 & 202 & 5390 & 53.124371 & -27.851633 & 0.16 & 0 & 2.81 & 0.35 & $3.700(1)$ & 44.55 \\
\hline 189 & 95 & 13855 & 53.124493 & -27.740086 & 0.69 & 0 & $<0.50$ & 0.81 & $0.076(2)$ & 40.27 \\
\hline 191 & 39 & 12354 & 53.124916 & -27.758301 & 0.08 & 0 & 15.57 & 8.44 & $1.218(1)$ & 44.06 \\
\hline 192 & 116 & 13853 & 53.124950 & -27.734684 & 0.90 & 0 & 0.89 & 0.61 & $0.076(2)$ & 40.1 \\
\hline 193 & 78 & 12489 & 53.125252 & -27.756536 & 0.04 & 0 & 2.75 & 1.94 & 0.960 (1) & 43.06 \\
\hline 195 & 38 & 12880 & 53.125900 & -27.751274 & 0.11 & 0 & 9.04 & 6.23 & $0.738(1)$ & 43.3 \\
\hline 196 & 516 & 9791 & 53.130642 & -27.790258 & 0.19 & 0 & $<0.42$ & 0.08 & 0.665 (1) & 41.91 \\
\hline 197 & 563 & 11272 & 53.131081 & -27.773107 & 0.23 & 0 & $<0.37$ & 0.07 & $2.223(1)$ & 42.64 \\
\hline 198 & 574 & 8217 & 53.131554 & -27.814941 & 0.47 & 0 & $<0.34$ & 0.09 & $1.879(6)$ & 42.56 \\
\hline 200 & $37 *$ & 16661 & 53.133640 & -27.698654 & 0.16 & 0 & 2.20 & 0.64 & $1.393(3)$ & 43.34 \\
\hline 201 & 515 & 70195 & 53.134022 & -27.781019 & 0.27 & $0^{m}$ & 1.23 & 0.11 & $2.98(1.9-4.6)$ & 43.9 \\
\hline 202 & $220 \mathrm{a}$ & 70404 & 53.136291 & -27.864195 & 0.57 & 0 & 0.75 & 0.35 & $1.42(1.3-1.6$ & 42.9 \\
\hline 204 & $36 \mathrm{a}$ & 11970 & 53.137573 & -27.763176 & 0.06 & 0 & 3.09 & 1.14 & $0.953(3)$ & 43.11 \\
\hline 205 & $217 \mathrm{a}$ & 70429 & 53.137924 & -27.868242 & 0.57 & $0^{k, m, r}$ & $<0.50$ & 0.19 & $>3.0$ & $>43.68$ \\
\hline 206 & 265 & 15927 & 53.138687 & -27.709997 & 0.22 & $0^{m}$ & 3.24 & 0.25 & $1.213(6)$ & 43.45 \\
\hline 207 & $514 *$ & 14880 & 53.139412 & -27.720207 & 0.34 & 0 & 0.93 & 0.20 & $0.103(5)$ & 40.4 \\
\hline 208 & $86 a$ & 12616 & 53.140991 & -27.755701 & 0.20 & 0 & $<0.48$ & 0.11 & $1.894(3)$ & 42.75 \\
\hline 209 & $513 a$ & 70289 & 53.141506 & -27.816603 & 0.92 & $0^{m}$ & 0.64 & $<0.06$ & $2.66(1.75-4.3)$ & 43.58 \\
\hline 210 & $579 \mathrm{~b}$ & 7316 & 53.141880 & -27.827168 & 0.00 & 0 & $<0.38$ & 0.08 & $0.832(3)$ & 41.59 \\
\hline 212 & 512 & 14262 & 53.143093 & -27.730581 & 0.64 & 0 & 0.72 & 0.20 & $0.668(1)$ & 42.11 \\
\hline 214 & $34 \mathrm{a}$ & 921 & 53.145634 & -27.919773 & 0.23 & 0 & 4.12 & 1.66 & 0.839 (1) & 43.1 \\
\hline 216 & 171 & 13946 & 53.146271 & -27.736269 & 0.07 & 0 & $<0.49$ & 0.14 & $0.839(2)$ & 41.82 \\
\hline 218 & 148 & 2818 & 53.146698 & -27.888338 & 0.36 & $0^{m}$ & 2.68 & 0.44 & $2.06(1.2-2.75)$ & 43.85 \\
\hline 219 & 901 & 7814 & 53.148827 & -27.821100 & 0.06 & 0 & 0.60 & 0.06 & $2.578(1)$ & 43.43 \\
\hline 221 & 100 & 8231 & 53.149887 & -27.814003 & 0.16 & 0 & 0.38 & 0.23 & $1.309(1)$ & 42.52 \\
\hline 222 & $239 \mathrm{a}$ & 4993 & 53.150711 & -27.857361 & 0.15 & 0 & $<0.39$ & 0.21 & $1.613(3)$ & 42.75 \\
\hline 223 & 609 & 5988 & 53.150658 & -27.843628 & 0.20 & 0 & 0.80 & $<0.10$ & $1.608(3)$ & 43.53 \\
\hline 224 & $577 \mathrm{a}$ & 7347 & 53.150726 & -27.825510 & 0.58 & 0 & $<0.34$ & 0.13 & 0.547 (1) & 41.35 \\
\hline 226 & $511 \mathrm{c}$ & 11049 & 53.152779 & -27.775293 & 0.40 & 0 & $<0.46$ & 0.13 & 0.999 (2) & 42.05 \\
\hline 227 & 33 & 13964 & 53.152977 & -27.735123 & 0.16 & 0 & 9.98 & 4.40 & 0.665 (2) & 43.24 \\
\hline 230 & 31 & 4119 & 53.157345 & -27.870085 & 0.13 & 0 & 10.78 & 6.45 & $1.603(1)$ & 44.18 \\
\hline 231 & 555 & 3010 & 53.158051 & -27.885572 & 0.24 & $0^{m}$ & $<0.46$ & 0.09 & $2.73(2.3-3.1)$ & 42.86 \\
\hline 233 & 79 & 11237 & 53.158451 & -27.773975 & 0.11 & $0^{m}$ & 1.85 & 0.95 & $>0.50$ & $>42.22$ \\
\hline 235 & 210 & 461 & 53.159641 & -27.931435 & 0.47 & $0^{m}$ & $<0.94$ & 0.37 & $1.61(1.2-2.25)$ & 43.12 \\
\hline 236 & $567 \mathrm{a}$ & 9696 & 53.161564 & -27.792257 & 0.19 & 0 & $<0.35$ & 0.09 & $0.458(5)$ & 41 \\
\hline 237 & 510 & 5099 & 53.161503 & -27.856007 & 0.28 & $0^{m}$ & 0.82 & 0.13 & $2.97(2.8-3.7)$ & 43.72 \\
\hline 238 & $582 \mathrm{~b}$ & 6757 & 53.161751 & -27.832291 & 0.12 & 0 & $<0.33$ & 0.07 & $0.242(3)$ & 40.92 \\
\hline 241 & $201 \mathrm{a}$ & 70116 & 53.162647 & -27.744331 & 0.59 & 0 & 2.18 & 0.52 & $2.52(1.5-4.0)$ & 43.96 \\
\hline 242 & 28 & 11701 & 53.162861 & -27.767160 & 0.12 & 0 & 3.24 & 0.90 & $1.216(1)$ & 43.38 \\
\hline 243 & 605 & 8625 & 53.163220 & -27.808992 & 0.32 & $0^{m}$ & 0.52 & $<0.04$ & $2.470(7)$ & 43.44 \\
\hline 244 & 586 & 6047 & 53.164421 & -27.842169 & 0.57 & 0 & 0.23 & 0.13 & $0.580(1)$ & 41.46 \\
\hline 245 & 27 & 8273 & 53.165272 & -27.814062 & 0.23 & 0 & 6.34 & 0.85 & $3.064(1)$ & 44.63 \\
\hline 246 & 26 & 11588 & 53.165394 & -27.769762 & 0.45 & 0 & 3.00 & 0.94 & $1.552(7)$ & 43.6 \\
\hline 247 & 25 & 540 & 53.170151 & -27.929647 & 0.14 & 0 & 7.53 & 0.62 & $0.625(1)$ & 43.11 \\
\hline 249 & $611 \mathrm{a}$ & 14708 & 53.173809 & -27.724491 & 0.65 & 0 & 1.05 & $<0.09$ & $0.979^{v}(1)$ & 42.95 \\
\hline 250 & 23 & 14158 & 53.174454 & -27.733299 & 0.27 & 0 & 3.16 & 1.72 & $2.576(6)$ & 44.12 \\
\hline 251 & 24 & 4302 & 53.174389 & -27.867353 & 0.23 & 0 & 4.37 & 1.83 & $3.610(1)$ & 44.59 \\
\hline 254 & 91 & 10429 & 53.178478 & -27.784033 & 0.13 & 0 & 1.84 & 0.70 & $3.193(1)$ & 44.11 \\
\hline 255 & 256 & 8374 & 53.179325 & -27.812527 & 0.20 & 0 & 2.73 & 0.10 & $1.730(7)$ & 43.76 \\
\hline 256 & 22 & 7822 & 53.180149 & -27.820604 & 0.05 & 0 & 7.36 & 3.97 & $1.920(1)$ & 44.19 \\
\hline 258 & $94 \mathrm{c}$ & 11006 & 53.183357 & -27.776384 & $0.70^{\dagger}$ & 0 & 0.70 & 0.60 & $2.688(1)$ & 43.51 \\
\hline
\end{tabular}


M. Brusa et al.: Black hole growth and starburst activity at $z=0.6-4$ in the Chandra Deep Field South, Online Material $p 3$

Table 1. continued.

\begin{tabular}{|c|c|c|c|c|c|c|c|c|c|c|}
\hline AID & XID & OID & $\begin{array}{c}\text { RA } \\
\text { J2000 }\end{array}$ & $\begin{array}{c}\text { Dec } \\
\text { J2000 }\end{array}$ & $\begin{array}{r}\text { dist } \\
\operatorname{arcsec}\end{array}$ & FLAG & $\begin{array}{c}S_{2-10} \mathrm{keV} \\
10^{-15} \mathrm{cgs}\end{array}$ & $\begin{array}{c}S_{0.5-2} \mathrm{keV} \\
10^{-15} \mathrm{cgs}\end{array}$ & $\overline{\text { Redshift }}$ & $\begin{array}{l}\log \left(L_{X}\right) \\
\text { erg s }^{-1}\end{array}$ \\
\hline 259 & $132 *$ & 1203 & 53.183304 & -27.915039 & 0.89 & 0 & 0.90 & 0.27 & $0.908(1)$ & 42.53 \\
\hline 260 & $98 \mathrm{a}$ & 4578 & 53.184448 & -27.861420 & 0.19 & 0 & $<0.45$ & 0.53 & $0.279(1)$ & 41.3 \\
\hline 261 & 21 & 3323 & 53.184639 & -27.880918 & 0.27 & 0 & 0.86 & 0.58 & $3.471(1)$ & 43.84 \\
\hline 262 & 573 & 8741 & 53.185150 & -27.805275 & 0.35 & 0 & 0.25 & 0.14 & $0.414(1)$ & 41.16 \\
\hline 263 & $20 \mathrm{a}$ & 7260 & 53.185230 & -27.827835 & 0.19 & 0 & 4.79 & 0.64 & $1.016(1)$ & 43.39 \\
\hline 264 & 85 & 8543 & 53.185833 & -27.809969 & 0.23 & 0 & 1.45 & 0.52 & $2.593(1)$ & 43.81 \\
\hline 266 & 646 & 9856 & 53.187954 & -27.789999 & 0.40 & 0 & $<0.34$ & 0.10 & $0.438(1)$ & 41.02 \\
\hline 268 & $147 *$ & 11062 & 53.193100 & -27.775555 & 0.11 & $0^{m}$ & 5.06 & 0.21 & $1.220(3)$ & 43.68 \\
\hline 269 & 170 & 1633 & 53.193371 & -27.903877 & 0.31 & 0 & 1.34 & 0.25 & 0.664 (1) & 42.38 \\
\hline 272 & 146 & 2532 & 53.196079 & -27.892647 & 0.25 & $0^{m}$ & 2.52 & 0.52 & $2.74(1.25-3.6)$ & 44.11 \\
\hline 273 & 592 & 4564 & 53.196575 & -27.863205 & 0.42 & 0 & $<0.55$ & 0.10 & $1.114(3)$ & 42.34 \\
\hline 276 & 184 & 3047 & 53.200737 & -27.882391 & 0.12 & 0 & 1.68 & 0.18 & 0.667 (1) & 43.15 \\
\hline 277 & $17 \mathrm{a}$ & 1059 & 53.204872 & -27.917955 & 0.44 & 0 & 2.15 & 0.86 & $2.02(1.4-2.75)$ & 43.72 \\
\hline 281 & 159 & 3320 & 53.209366 & -27.881090 & 0.33 & 0 & 8.19 & 2.67 & 3.470 (4) & 44.84 \\
\hline 283 & 508 & 70435 & 53.215118 & -27.870249 & 0.20 & $0^{k, m}$ & 1.09 & 0.15 & $>1.50$ & $>43.15$ \\
\hline 286 & 15 & 5062 & 53.220356 & -27.855511 & 0.27 & 0 & 6.41 & 3.15 & $1.227(1)$ & 43.68 \\
\hline 292 & $553 a$ & 2750 & 53.236080 & -27.887917 & 0.75 & 0 & $<0.78$ & 0.25 & $0.366(1)$ & 41.23 \\
\hline 300 & 240 & 4751 & 53.245850 & -27.861208 & 1.03 & $0^{m}$ & $<0.95$ & 0.27 & $0.16(0.0-2.0)$ & 40.47 \\
\hline 70 & 537 & 70340 & 53.040970 & -27.837717 & 0.52 & 1 & $<0.48$ & 0.11 & $>5.00$ & $>43.5$ \\
\hline 81 & 236 & 6685 & 53.047676 & -27.834925 & 0.39 & 1 & 0.46 & 0.24 & $1.920(4)$ & 42.26 \\
\hline 89 & 257 & 70286 & 53.055836 & -27.815449 & 0.48 & 1 & 1.28 & 0.08 & $2.27(1.0-4.0)$ & 43.68 \\
\hline 104 & 587 & 70357 & 53.063774 & -27.843531 & 1.08 & 1 & 0.31 & 0.13 & $2.28(1.6-3.8)$ & 43 \\
\hline $128^{a}$ & 254 & 70139 & 53.082344 & -27.755135 & 0.61 & 1 & 1.60 & 0.05 & $2.28(1.4-4.2)$ & 43.82 \\
\hline 135 & 96 & 80001 & 53.086806 & -27.872953 & 0.21 & 1 & 0.90 & 0.33 & -1.00 & -1 \\
\hline 140 & 902 & 70380 & 53.091476 & -27.853201 & 0.75 & 1 & $<0.44$ & 0.08 & $>1.30$ & $>42.08$ \\
\hline 144 & 570 & 70251 & 53.093704 & -27.801287 & 0.32 & 1 & $<0.35$ & 0.17 & $2.84(0.4-4.4)$ & 43.15 \\
\hline 232 & 557 & 70091 & 53.158222 & -27.733713 & 0.60 & $1^{r}$ & $<0.43$ & 0.09 & $>3.80$ & $>43.17$ \\
\hline 74 & $\ldots$ & 11122 & 53.044952 & -27.774378 & 0.21 & 2 & $<0.44$ & 0.10 & $1.607(2)$ & 42.34 \\
\hline 85 & $\ldots$ & 16795 & 53.052723 & -27.696955 & 0.18 & 2 & $<0.83$ & 0.08 & $1.78(1.4-2.3)$ & 42.71 \\
\hline 97 & $\ldots$ & 13053 & 53.060192 & -27.749054 & 0.52 & 2 & $<0.49$ & 0.04 & $0.738(2)$ & 41.6 \\
\hline 107 & $\ldots$ & 11065 & 53.065842 & -27.775141 & 0.63 & 2 & $<0.37$ & $<0.06$ & $1.023(2)$ & 42.34 \\
\hline $119^{a}$ & $\ldots$ & 8977 & 53.073456 & -27.803286 & 1.11 & 2 & 0.27 & $<0.08$ & $0.735(3)$ & 41.83 \\
\hline 132 & $\ldots$ & 11695 & 53.084652 & -27.765228 & 0.65 & 2 & $<0.36$ & $0.14^{f}$ & $0.231(2)$ & 41.74 \\
\hline 138 & $\ldots$ & 70198 & 53.090870 & -27.782350 & 0.94 & 2 & 0.29 & $<0.03$ & $>1.80$ & $>42.85$ \\
\hline 142 & $\ldots$ & 70307 & 53.092407 & -27.826687 & 0.69 & 2 & $<0.43$ & 0.06 & $>1.75$ & $>42.39$ \\
\hline 148 & $\ldots$ & 13552 & 53.094093 & -27.740505 & 0.64 & 2 & $<0.49$ & $0.19^{f}$ & $0.738(2)$ & 42.25 \\
\hline 165 & $\ldots$ & 70449 & 53.105164 & -27.875032 & 0.81 & 2 & $<0.37$ & 0.06 & $2.92(1.7-4.7)$ & 42.81 \\
\hline 178 & $\ldots$ & 2768 & 53.112713 & -27.888447 & 0.27 & 2 & $<0.33$ & 0.08 & $1.103(3)$ & 41.84 \\
\hline 185 & $\ldots$ & 4402 & 53.122162 & -27.865400 & 0.23 & 2 & $<0.47$ & 0.07 & $0.511(7)$ & 41.18 \\
\hline 190 & $\ldots$ & 3290 & 53.124699 & -27.881210 & 0.31 & 2 & $<0.45$ & 0.06 & $1.017(3)$ & 41.86 \\
\hline 199 & $\ldots$ & 13111 & 53.133503 & -27.747704 & 0.29 & 2 & $<0.44$ & $0.17^{f}$ & 0.895 (2) & 41.8 \\
\hline 203 & $\ldots$ & 17284 & 53.137443 & -27.688057 & 0.59 & 2 & $<0.96$ & 0.05 & $1.071(4)$ & 42.32 \\
\hline $215^{p}$ & $\ldots$ & 7453 & 53.146000 & -27.825754 & 0.92 & 2 & $<0.19$ & 0.05 & $2.22(1.7-2.6)$ & 42.36 \\
\hline 217 & $\ldots$ & 70437 & 53.146461 & -27.870947 & 0.45 & $2^{r}$ & $<0.45$ & $0.18^{f}$ & $>4.42$ & $>43.49$ \\
\hline $225^{p}$ & $\cdots$ & 7443 & 53.151447 & -27.825872 & 0.28 & $2^{s}$ & $<0.34$ & 0.06 & 0.70 & 41.34 \\
\hline $239^{p}$ & $\ldots$ & 3743 & 53.161980 & -27.875204 & 1.57 & 2 & $<0.34$ & 0.04 & $2.01(1.0-2.6)$ & 42.45 \\
\hline 248 & $\ldots$ & 9957 & 53.172524 & -27.788107 & 1.25 & 2 & $<0.33$ & 0.04 & $0.622(7)$ & 41.24 \\
\hline 257 & $\ldots$ & 8053 & 53.181049 & -27.817164 & 0.39 & 2 & $<0.27$ & 0.05 & $1.603(4)$ & 42.29 \\
\hline 265 & $\cdots$ & 1263 & 53.187576 & -27.910996 & 1.02 & 2 & $<0.68$ & 0.16 & $0.458(7)$ & 41.26 \\
\hline 267 & $\cdots$ & 564 & 53.190334 & -27.926205 & 0.46 & 2 & 0.24 & 0.12 & $0.102(3)$ & 39.81 \\
\hline
\end{tabular}


M. Brusa et al.: Black hole growth and starburst activity at $z=0.6-4$ in the Chandra Deep Field South, Online Material $p 4$

Table 1. continued.

\begin{tabular}{|c|c|c|c|c|c|c|c|c|c|c|}
\hline AID & XID & OID & $\begin{array}{c}\text { RA } \\
\text { J2000 }\end{array}$ & $\begin{array}{c}\text { Dec } \\
\text { J2000 }\end{array}$ & $\begin{array}{r}\text { dist } \\
\operatorname{arcsec}\end{array}$ & FLAG & $\begin{array}{c}S_{2-10} \mathrm{keV} \\
10^{-15} \mathrm{cgs}\end{array}$ & $\begin{array}{r}S_{0.5-2} \mathrm{keV} \\
10^{-15} \mathrm{cgs}\end{array}$ & Redshift & $\begin{array}{l}\log \left(L_{X}\right) \\
\text { erg s }^{-1}\end{array}$ \\
\hline $183^{p}$ & . & 70241 & 53.120380 & -27.798939 & $0.73^{\dagger}$ & $2^{a a}$ & $<0.23$ & 0.04 & $2.94(1.3-3.60)$ & 42.62 \\
\hline & & 9269 & 53.1198 & -27.798738 & 1.04 & 2 & $<0.23$ & 0.04 & & \\
\hline 186 & 264 & 70403 & 53.123787 & -27.862678 & $0.31^{\dagger}$ & 1 & 1.77 & 0.06 & $2.47(1.4-5.5)$ & 43.95 \\
\hline & & 4606 & 53.123939 & -27.863092 & 1.54 & 0 & 1.77 & 0.06 & &.. \\
\hline 282 & $\ldots$ & $\ldots$ & $\ldots$ & $\ldots$ & $\ldots$ & - & 1.50 & $<0.12$ & -1.00 & \\
\hline
\end{tabular}

Notes: AID = X-ray identifier from A03; XID = X-ray identifier from S04, OID = MUSIC identifier from Santini et al. (2009).

$a$ : X-ray positions corrected from that reported in A03 (by visual inspection of the event file); $\uparrow$ tentative X-ray to IRAC association; in this case, also a "secondary" candidate counterpart is reported; $\ddagger$ optical/IRAC counterpart not associated. Legenda for the FLAG column: 0 - counterpart published in S04; 1 - counterpart different from the one published in S04; 2 - new published counterparts (sources detected only in A03). Notes on single sources: ${ }^{k}$ : source published in Koekemoer et al. (2004); ${ }^{m}$ : source published in Mainieri et al. (2005); ${ }^{r}$ : source published in Rodighiero et al. (2007); ${ }^{a a}$ : source published by Alonso-Herrero et al. (2006); ${ }^{\text {s: }}$ this source is listed as counterpart "b" of XID 557 in S04 (577b); ${ }^{f}$ : flux in the $0.5-8 \mathrm{keV}$ band; ${ }^{p}$ : possible spurious source (see discussion in Sect. 2.1). The redshift column report the photometric or spectroscopic redshift. When a spectroscopic redshift is present, the relative source catalog is reported, as follow: (1) CXO-CDFS (S04); (2) K20 (Mignoli et al. 2005); (3) GOODS-FORS2 (various releases); (4) GOODS-VIMOS (Popesso et al. 2008); (5) COMBO-17 (Wolf et al. 2003); (6) GMASS; (7) others: VVDS, Daddi, TrzK, LCIRS ast0612152 Otherwise, the redshift is from SED fitting. (CCC): photometric redshift soultion preferred to the low-quality spectroscopic redshift available; ${ }^{v}$ : this object has a different redshift solution from the VIMOS observation, $z=0.798$ (Popesso et al. 2008). 
M. Brusa et al.: Black hole growth and starburst activity at $z=0.6-4$ in the Chandra Deep Field South, Online Material $p 5$

Table 2. Multiwavelength photometry for counterparts to CDFS X-ray sources.

\begin{tabular}{|c|c|c|c|c|c|c|c|c|c|c|c|c|c|}
\hline 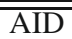 & 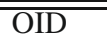 & $\overline{c b}$ & $\bar{v}$ & $\bar{i}$ & $z$ & $j$ & $\overline{\bar{h}}$ & $\overline{\bar{k}}$ & $S(3.6)$ & $S(4.5)$ & $S(5.8)$ & $\overline{S S(8.0)}$ & $\overline{C S(24)}$ \\
\hline 51 & 13272 & 26.51 & 26.27 & 25.53 & 25.17 & 23.99 & 23.24 & 23.06 & 22.33 & 22.15 & 22.16 & 22.44 & $>20.93$ \\
\hline 53 & 12222 & 25.53 & 23.12 & 21.63 & 21.13 & 20.61 & 20.07 & 19.67 & 19.75 & 20.16 & 20.44 & 21.64 & $>22.46$ \\
\hline 67 & 70103 & 28.06 & 28.03 & 26.72 & 26.78 & $>26.07$ & 23.76 & 23.67 & 22.60 & 22.43 & 22.50 & 22.83 & 22.56 \\
\hline 71 & 70051 & 26.28 & 24.47 & 23.19 & 22.51 & 22.38 & 21.38 & 20.30 & 19.31 & 19.57 & 19.23 & 18.90 & 17.38 \\
\hline 73 & 15717 & 21.81 & 19.97 & 19.06 & 18.68 & 18.17 & & 17.58 & 18.04 & 18.30 & 18.68 & 19.10 & 18.86 \\
\hline 75 & 14349 & 23.14 & 22.60 & 21.62 & 20.97 & 20.66 & 20.10 & 19.76 & 19.09 & 19.43 & 19.75 & 20.00 & 18.55 \\
\hline 76 & 13849 & 23.70 & 22.96 & 22.38 & 22.39 & 21.66 & 21.41 & 20.93 & 20.02 & 19.65 & 19.33 & 19.11 & 17.91 \\
\hline 77 & 8168 & 27.75 & 26.92 & 26.01 & 25.06 & 24.04 & 23.07 & 22.08 & 21.56 & 21.42 & 20.97 & 20.67 & 18.95 \\
\hline 80 & 10619 & 24.25 & 22.74 & 21.66 & 21.30 & 20.69 & 20.49 & 20.24 & 20.32 & 20.64 & 20.78 & 21.25 & 20.16 \\
\hline 82 & 11180 & 26.89 & 26.43 & 25.57 & 25.08 & 23.55 & 23.19 & 23.11 & 21.96 & 21.28 & 21.01 & 20.47 & 19.00 \\
\hline 83 & 12281 & 24.59 & 22.47 & 21.15 & 20.74 & 20.31 & 19.87 & 19.48 & 19.61 & 20.05 & 20.27 & 20.94 & 20.20 \\
\hline 84 & 11285 & 24.81 & 23.67 & 22.60 & 21.92 & 21.14 & 20.80 & 20.31 & 19.69 & 19.85 & 20.23 & 20.30 & 18.03 \\
\hline 88 & 15626 & 21.83 & 20.92 & 20.06 & 19.84 & 19.25 & $\ldots$ & 18.63 & 18.60 & 18.85 & 18.74 & 18.81 & 16.82 \\
\hline 90 & 12434 & 25.23 & 24.39 & 23.32 & 22.48 & 21.33 & 20.64 & 20.04 & 19.54 & 19.49 & 19.90 & 20.58 & 19.48 \\
\hline 91 & 15382 & 23.41 & 22.36 & 21.27 & 20.83 & 20.04 & & 19.22 & 19.15 & 19.50 & 19.48 & 19.39 & 17.74 \\
\hline 93 & 70323 & $>28.37$ & 27.06 & 26.96 & 26.02 & 23.52 & 21.71 & 21.29 & 20.19 & 20.05 & 19.76 & 19.84 & 21.44 \\
\hline 94 & 5434 & 23.95 & 23.10 & 22.05 & 21.57 & 21.08 & 20.65 & 20.28 & 20.14 & 20.87 & 20.36 & 20.34 & 18.93 \\
\hline 95 & 16040 & 24.79 & 25.04 & 24.60 & 24.50 & 24.22 & & 23.48 & 22.42 & 21.82 & 21.59 & 21.65 & 19.32 \\
\hline 96 & 5281 & 24.34 & 23.94 & 23.38 & 22.86 & 21.68 & 21.26 & 21.05 & 22.95 & 20.29 & 20.64 & 20.90 & 19.25 \\
\hline 99 & 14104 & 27.04 & 26.66 & 25.61 & 25.13 & 24.02 & 23.10 & 22.75 & 21.68 & 21.51 & 21.60 & 21.83 & 20.08 \\
\hline 100 & 5440 & $>29.21$ & 27.35 & 25.96 & 24.91 & 23.19 & 22.75 & 22.03 & 21.09 & 21.64 & 21.27 & 21.42 & 20.54 \\
\hline 102 & 16094 & 24.88 & 23.67 & 22.95 & 22.15 & 20.30 & $\ldots$ & 19.66 & 19.08 & 18.95 & 19.05 & 19.57 & 19.87 \\
\hline 103 & 4878 & 23.80 & 22.28 & 20.93 & 20.47 & 19.89 & $\ldots$ & 19.14 & 19.14 & 20.21 & 19.60 & 20.11 & 19.69 \\
\hline 106 & 2654 & 24.75 & 23.26 & 22.17 & 21.73 & 21.12 & $\ldots$ & 20.29 & 19.98 & 20.83 & 20.45 & 20.62 & 18.23 \\
\hline 111 & 4384 & 26.42 & 25.94 & 25.74 & 25.47 & 24.66 & & 23.92 & 23.16 & 24.92 & 22.79 & 22.91 & 21.58 \\
\hline 112 & 10944 & 27.25 & 26.63 & 26.17 & 25.89 & $>26.74$ & 25.03 & $>26.08$ & 24.56 & 23.95 & 23.91 & 23.12 & $>21.14$ \\
\hline 113 & 14774 & 24.86 & 22.96 & 22.02 & 21.74 & 21.27 & $\ldots$ & 20.91 & 21.24 & 21.55 & 21.80 & 22.36 & $>21.11$ \\
\hline 114 & 6606 & 26.18 & 24.35 & 22.92 & 22.53 & 21.95 & 21.67 & 21.43 & 21.47 & 22.03 & 22.23 & 22.67 & $>21.12$ \\
\hline 115 & 7393 & 22.04 & 20.30 & 19.47 & 19.13 & 18.64 & 18.22 & 18.08 & 18.49 & 18.76 & 19.13 & 18.87 & 18.09 \\
\hline 116 & 17038 & 27.45 & 26.59 & 26.11 & 25.64 & 23.96 & $\ldots$ & 22.82 & 21.92 & 21.57 & 21.42 & 21.61 & 19.49 \\
\hline 117 & 15207 & 23.11 & 21.76 & 20.86 & 20.58 & 20.01 & $\ldots$ & 19.36 & 19.31 & 19.41 & 19.35 & 19.09 & 16.05 \\
\hline 118 & 3920 & 24.97 & 23.87 & 22.65 & 21.80 & 21.03 & & 19.93 & 19.46 & 19.86 & 19.70 & 19.52 & 17.89 \\
\hline 121 & 9792 & 24.14 & 21.61 & 19.95 & 19.35 & 18.55 & 18.08 & 17.74 & 17.60 & 18.18 & 18.37 & 19.05 & 20.10 \\
\hline 122 & 3533 & 24.51 & 24.36 & 24.25 & 24.30 & 24.01 & . & 23.41 & 22.68 & 22.55 & 22.19 & 22.34 & 21.46 \\
\hline 123 & 5551 & 24.92 & 24.32 & 23.35 & 22.60 & 21.18 & 20.75 & 20.39 & 19.80 & 19.87 & 20.13 & 20.69 & 19.37 \\
\hline 124 & 13866 & 24.91 & 23.92 & 22.74 & 22.08 & 21.37 & 20.82 & 20.37 & 20.26 & 20.57 & 20.95 & 21.30 & 19.88 \\
\hline 125 & 4835 & 27.75 & 25.68 & 25.20 & 25.08 & 24.31 & $\ldots$ & 22.50 & 21.75 & 21.74 & 21.57 & 21.25 & 20.01 \\
\hline 126 & 9279 & 26.46 & 24.92 & 23.77 & 23.45 & 22.83 & 22.71 & 22.49 & 22.23 & 22.33 & 22.03 & 21.63 & 20.08 \\
\hline 129 & 17067 & 21.04 & 19.60 & 18.97 & 18.73 & 18.34 & & 17.95 & 18.28 & 18.03 & 17.84 & 7.18 & 15.15 \\
\hline 130 & 70052 & 27.65 & 28.44 & 26.86 & 26.25 & 24.86 & 23.57 & 22.97 & 23.05 & 23.01 & 22.51 & 23.18 & $>21.01$ \\
\hline 131 & 13250 & 25.95 & 25.32 & 24.68 & 24.30 & 23.40 & 22.81 & 22.34 & 21.35 & 21.03 & 20.73 & 20.27 & 17.63 \\
\hline 133 & 70044 & $>28.56$ & $>28.86$ & $>28.07$ & $>27.96$ & $>26.00$ & $>25.06$ & $>25.25$ & 23.49 & 23.05 & 22.56 & 22.15 & $>21.01$ \\
\hline 134 & 9723 & 24.95 & 23.31 & 22.11 & 21.62 & 21.02 & 20.24 & 19.88 & 19.73 & 20.15 & 20.13 & 20.38 & 19.15 \\
\hline 136 & 489 & 25.55 & 25.01 & 24.67 & 24.45 & 23.81 & $\ldots$ & 23.37 & 22.75 & 23.13 & 22.16 & 22.68 & 20.51 \\
\hline 137 & 15948 & 23.43 & 22.56 & 21.53 & 21.16 & 20.50 & 19.97 & 19.86 & 19.81 & 20.28 & 20.32 & 20.76 & 18.71 \\
\hline 139 & 10384 & 23.77 & 22.27 & 20.92 & 20.46 & 19.76 & 19.25 & 19.02 & 18.94 & 19.43 & 19.56 & 20.11 & 18.62 \\
\hline 141 & 70249 & 27.27 & 28.91 & 28.08 & $>27.96$ & 24.60 & 23.59 & 22.76 & 21.30 & 21.00 & 20.47 & 20.47 & 18.21 \\
\hline 143 & 12146 & 27.70 & 27.70 & 26.87 & 26.57 & 25.85 & 25.15 & 24.75 & 23.78 & 23.55 & 23.36 & 23.74 & $>21.50$ \\
\hline 145 & 11723 & 25.18 & 25.09 & 24.57 & 24.32 & 23.34 & 22.91 & 22.33 & 21.11 & 20.54 & 20.07 & 19.61 & 18.64 \\
\hline 146 & 7031 & 26.18 & 24.12 & 22.62 & 22.03 & 21.09 & 20.48 & 19.92 & 19.72 & 20.26 & 20.30 & 20.74 & 19.02 \\
\hline 149 & 3781 & 21.21 & 20.41 & 19.99 & 19.83 & 19.67 & $\ldots$ & 19.46 & 20.07 & 20.89 & 20.93 & 20.14 & 18.77 \\
\hline 151 & 11854 & 24.65 & 23.95 & 23.13 & 22.33 & 21.76 & 21.25 & 20.93 & 20.34 & 20.36 & 20.78 & 20.76 & 18.81 \\
\hline 152 & 8029 & 20.80 & 20.10 & 19.86 & 19.80 & 19.62 & 19.68 & 19.57 & 20.15 & 20.39 & 20.80 & 19.85 & 19.03 \\
\hline 154 & 15419 & 25.14 & 24.68 & 24.47 & 24.17 & 23.25 & 22.27 & 21.86 & 21.28 & 21.07 & 20.96 & 21.28 & 20.05 \\
\hline 155 & 17077 & 23.34 & 21.66 & 20.54 & 20.11 & 19.58 & 19.08 & 18.80 & 19.06 & 19.32 & 19.48 & 19.89 & 19.31 \\
\hline 157 & 1780 & 24.77 & 22.80 & 21.52 & 21.10 & 20.39 & $\ldots$ & 19.73 & 19.67 & 20.70 & 20.33 & 20.91 & 19.84 \\
\hline 158 & 10250 & 22.11 & 21.06 & 20.59 & 20.32 & 20.10 & 19.48 & 19.58 & 19.95 & 19.95 & 20.33 & 19.18 & 18.13 \\
\hline 159 & 371 & 25.60 & 24.95 & 23.90 & 23.20 & 22.30 & $\ldots$ & 21.35 & 20.74 & 21.03 & 20.97 & 21.40 & 20.12 \\
\hline 160 & 6640 & 25.80 & 25.43 & 24.41 & 23.97 & 23.34 & 23.02 & 22.80 & 22.34 & 22.61 & 22.52 & 22.36 & $>22.28$ \\
\hline 162 & 5832 & 24.79 & 24.24 & 23.25 & 22.67 & 22.27 & 22.13 & 21.89 & 21.48 & 21.85 & 22.02 & 22.08 & 20.24 \\
\hline 163 & 16224 & 24.79 & 23.62 & 22.64 & 22.21 & 21.59 & 20.96 & 21.05 & 20.33 & 19.70 & 19.38 & 19.01 & 17.53 \\
\hline 164 & 1192 & 24.76 & 23.53 & 22.17 & 21.21 & 20.48 & $\cdots$ & 19.64 & 18.98 & 19.63 & 19.71 & 20.14 & 19.04 \\
\hline 166 & 15260 & 26.62 & 26.13 & 25.44 & 25.02 & 23.61 & 22.29 & 21.89 & 20.79 & 20.11 & 19.25 & 18.37 & 17.12 \\
\hline 167 & 7194 & 22.54 & 21.10 & 20.04 & 19.66 & 19.13 & 18.75 & 18.54 & 18.55 & 19.03 & 19.24 & 19.90 & 19.45 \\
\hline
\end{tabular}


M. Brusa et al.: Black hole growth and starburst activity at $z=0.6-4$ in the Chandra Deep Field South, Online Material $p 6$

Table 2. continued.

\begin{tabular}{|c|c|c|c|c|c|c|c|c|c|c|c|c|c|}
\hline AID & OID & $\bar{b}$ & $\bar{v}$ & $\bar{i}$ & $\bar{z}$ & $\bar{j}$ & $\bar{h}$ & $\bar{k}$ & $\overline{S(3.6)}$ & $S(4.5)$ & $S(5.8)$ & $\overline{S(8.0)}$ & $S(24)$ \\
\hline 170 & 70385 & $>28.60$ & 27.77 & 28.01 & 26.49 & 25.73 & $>25.26$ & 22.65 & 22.56 & 22.37 & 22.08 & 22.16 & $>21.39$ \\
\hline 171 & 978 & 24.19 & 23.73 & 23.00 & 22.55 & 22.09 & $\ldots$ & 21.77 & 21.25 & 21.63 & 21.51 & 21.75 & 19.48 \\
\hline 172 & 12747 & 27.75 & 26.61 & 26.07 & 25.95 & 25.05 & 23.99 & 23.42 & 23.01 & 22.78 & 22.54 & 21.68 & 21.72 \\
\hline 175 & 70168 & $>28.40$ & 28.20 & 26.54 & 25.97 & $>25.81$ & $>25.03$ & 24.03 & 22.92 & 23.00 & 22.04 & 21.77 & 20.63 \\
\hline 176 & 16792 & 24.39 & 23.08 & 21.87 & 21.44 & 20.81 & 20.23 & 19.94 & 19.49 & 19.59 & 19.40 & 19.20 & 17.43 \\
\hline 177 & 17452 & 19.40 & 19.31 & 19.13 & 19.00 & 18.77 & 18.68 & 18.41 & 17.51 & 17.21 & 17.03 & 16.89 & 16.56 \\
\hline 179 & 16831 & 24.05 & 22.81 & 21.62 & 21.31 & 20.91 & 20.33 & 20.10 & 19.65 & 19.56 & 19.26 & 18.96 & 17.50 \\
\hline 180 & 10464 & 25.14 & 24.78 & 24.40 & 24.18 & 23.33 & 22.73 & 22.69 & 21.68 & 21.55 & 21.30 & 21.59 & 19.04 \\
\hline 181 & 11248 & 25.13 & 23.17 & 21.70 & 21.15 & 20.60 & 19.72 & 19.70 & 19.63 & 20.11 & 20.30 & 20.92 & $>21.08$ \\
\hline 182 & 13977 & 20.50 & 18.85 & 18.18 & 17.87 & 17.52 & 17.34 & 17.19 & 17.79 & 18.06 & 18.67 & 18.98 & 19.81 \\
\hline 187 & 2637 & 29.66 & 27.59 & 26.94 & 26.47 & 25.32 & $\ldots$ & 22.99 & 21.73 & 21.62 & 21.26 & 21.55 & 20.22 \\
\hline 188 & 5390 & 27.52 & 25.05 & 24.53 & 24.62 & 24.50 & 23.44 & 22.49 & 21.91 & 21.81 & 21.62 & 21.48 & 19.20 \\
\hline 189 & 13855 & 17.57 & 16.86 & 16.45 & 16.30 & 16.01 & 15.87 & 15.92 & 16.14 & 16.41 & 15.97 & 13.86 & 14.06 \\
\hline 191 & 12354 & 21.15 & 21.19 & 21.12 & 21.07 & 20.84 & 20.60 & 20.20 & 19.39 & 19.08 & 18.94 & 18.62 & 17.33 \\
\hline 192 & 13853 & 18.16 & 17.39 & 16.95 & 16.76 & 16.45 & 16.28 & 16.32 & 16.90 & 16.96 & 16.68 & 14.73 & 14.64 \\
\hline 193 & 12489 & 23.45 & 22.87 & 22.23 & 21.74 & 21.11 & 20.82 & 20.21 & 19.78 & 19.82 & 19.89 & 19.73 & 17.49 \\
\hline 195 & 12880 & 22.61 & 22.10 & 21.72 & 21.51 & 21.27 & 21.22 & 20.93 & 20.64 & 20.68 & 20.52 & 20.56 & 18.60 \\
\hline 196 & 9791 & 22.57 & 21.90 & 21.24 & 21.08 & 20.68 & 20.14 & 20.06 & 20.22 & 20.62 & 20.50 & 20.79 & 18.60 \\
\hline 197 & 11272 & 24.37 & 23.68 & 23.21 & 22.92 & 22.06 & 21.07 & 20.81 & 20.33 & 20.01 & 19.61 & 19.27 & 17.66 \\
\hline 198 & 8217 & 25.90 & 25.43 & 25.21 & 24.87 & 23.70 & 23.15 & 22.86 & 21.30 & 21.00 & 20.85 & 21.21 & 19.50 \\
\hline 200 & 16661 & 24.71 & 24.17 & 23.53 & 23.10 & 22.43 & 21.85 & 21.50 & 20.68 & 20.33 & 20.07 & 20.31 & 17.72 \\
\hline 201 & 70195 & $>28.71$ & 27.02 & 26.89 & 26.14 & $>25.72$ & 23.61 & 23.34 & 22.36 & 22.39 & 21.71 & 21.33 & 20.16 \\
\hline 202 & 70404 & 24.53 & 23.79 & 23.00 & 22.43 & 21.45 & 20.87 & 20.44 & 19.47 & 19.68 & 19.69 & 19.96 & 20.07 \\
\hline 204 & 11970 & 24.27 & 23.06 & 21.89 & 21.26 & 20.36 & 19.88 & 19.22 & 19.07 & 19.26 & 19.48 & 19.57 & 17.54 \\
\hline 205 & 70429 & $>28.63$ & $>28.82$ & $>28.04$ & $>27.90$ & $>25.96$ & 25.41 & 23.86 & 23.27 & 22.98 & 22.49 & 22.48 & $>21.01$ \\
\hline 206 & 15927 & $>29.15$ & 27.19 & 25.75 & 24.63 & 23.45 & 22.60 & 22.41 & 22.10 & 21.52 & 21.18 & 20.60 & 20.09 \\
\hline 207 & 14880 & 18.78 & 17.48 & 16.90 & 16.62 & 16.27 & 15.94 & 16.09 & 17.56 & 17.12 & 17.60 & 17.51 & 17.94 \\
\hline 208 & 12616 & 26.53 & 25.76 & 25.39 & 25.09 & 24.16 & 23.69 & 23.10 & 22.78 & 22.45 & 22.39 & 22.37 & 21.36 \\
\hline 209 & 70289 & 27.46 & 27.06 & 26.68 & 26.22 & 25.43 & 23.60 & 22.91 & 21.70 & 21.43 & 21.02 & 21.08 & 19.36 \\
\hline 210 & 7316 & 27.33 & 25.14 & 23.60 & 22.89 & 22.26 & 21.74 & 21.34 & 21.18 & 21.64 & 21.84 & 22.39 & $>21.13$ \\
\hline 212 & 14262 & 24.61 & 22.79 & 21.49 & 21.07 & 20.51 & 20.23 & 19.95 & 20.48 & 20.38 & 20.60 & 21.20 & 20.29 \\
\hline 214 & 921 & 23.76 & 23.15 & 22.21 & 21.79 & 20.92 & & 20.23 & 20.14 & 20.36 & 20.20 & 20.07 & 18.18 \\
\hline 216 & 13946 & 25.11 & 24.49 & 23.86 & 23.54 & 22.43 & 21.99 & 21.44 & 21.83 & 20.58 & 20.53 & 21.02 & 18.50 \\
\hline 218 & 2818 & 28.54 & 28.18 & 27.03 & 26.06 & 24.61 & $\ldots$ & 23.45 & 22.42 & 22.25 & 21.84 & 21.67 & 20.02 \\
\hline 219 & 7814 & 25.68 & 25.39 & 25.00 & 24.88 & 23.81 & 22.36 & 22.01 & 21.16 & 20.75 & 20.51 & 19.77 & 17.00 \\
\hline 221 & 8231 & 22.42 & 22.23 & 22.00 & 21.64 & 21.44 & 21.09 & 20.95 & 20.48 & 20.51 & 20.88 & 20.91 & 19.17 \\
\hline 222 & 4993 & 25.97 & 25.36 & 24.43 & 23.91 & 22.99 & $\ldots$ & 21.73 & 20.81 & 20.70 & 20.86 & 21.14 & 18.99 \\
\hline 223 & 5988 & 29.38 & 28.02 & 26.81 & 25.84 & 23.68 & 22.86 & 22.39 & 21.48 & 21.57 & 21.49 & 21.97 & 21.16 \\
\hline 224 & 7347 & 22.77 & 21.48 & 20.62 & 20.24 & 19.67 & 19.05 & 18.92 & 18.86 & 19.11 & 19.05 & 18.74 & 16.81 \\
\hline 226 & 11049 & 24.99 & 23.88 & 22.69 & 22.13 & 21.51 & 21.20 & 20.77 & 20.52 & 20.83 & 21.09 & 21.12 & 19.12 \\
\hline 227 & 13964 & 24.43 & 23.18 & 22.05 & 21.64 & 21.06 & 20.74 & 20.41 & 20.96 & 20.38 & 20.38 & 20.48 & 19.08 \\
\hline 230 & 4119 & 25.15 & 24.72 & 24.08 & 23.52 & 21.68 & $\ldots$ & 20.59 & 19.56 & 19.17 & 18.57 & 17.99 & 16.44 \\
\hline 231 & 3010 & 26.86 & 25.91 & 25.96 & 25.76 & 24.52 & $\ldots$ & 23.45 & 22.45 & 22.34 & 22.15 & 22.13 & 20.36 \\
\hline 233 & 11237 & $>30.06$ & 28.70 & 27.60 & 26.48 & 24.91 & 23.77 & 22.98 & 22.04 & 21.79 & 21.30 & 20.62 & 19.35 \\
\hline 235 & 461 & 28.37 & 27.97 & 26.71 & 25.91 & 23.84 & $\cdots$ & 22.22 & 21.21 & 20.98 & 21.06 & 21.34 & 18.65 \\
\hline 236 & 9696 & 21.97 & 21.53 & 21.18 & 20.93 & 20.72 & 20.31 & 20.07 & 20.19 & 20.14 & 20.16 & 19.32 & 16.81 \\
\hline 237 & 5099 & 27.81 & 26.33 & 26.14 & 26.11 & 25.10 & $\ldots$ & 23.55 & 22.88 & 22.82 & 22.46 & 21.99 & 19.62 \\
\hline 238 & 6757 & 21.99 & 20.69 & 19.96 & 19.64 & 18.90 & 18.35 & 17.93 & 18.62 & 18.64 & 19.00 & 17.23 & 17.11 \\
\hline 241 & 70116 & 27.05 & 26.68 & 26.44 & 26.06 & $>25.89$ & 23.40 & 23.23 & 22.66 & 22.49 & 22.09 & 21.89 & $>21.85$ \\
\hline 242 & 11701 & 21.26 & 21.02 & 20.96 & 20.99 & 21.10 & 20.55 & 20.59 & 19.42 & 19.27 & 19.18 & 18.97 & 17.97 \\
\hline 243 & 8625 & $>29.64$ & 27.58 & 26.36 & 26.21 & 25.18 & 23.37 & 22.87 & 22.33 & 22.20 & 22.07 & 22.17 & $>22.61$ \\
\hline 244 & 6047 & 25.21 & 22.96 & 21.65 & 21.21 & 20.62 & 20.13 & 19.91 & 19.99 & 20.52 & 20.64 & 21.35 & $>21.48$ \\
\hline 245 & 8273 & 28.93 & 25.68 & 24.70 & 24.51 & 23.77 & 22.40 & 21.67 & 21.05 & 20.84 & 20.61 & 20.31 & 18.69 \\
\hline 246 & 11588 & 26.02 & 25.83 & 25.30 & 24.93 & 24.33 & 23.24 & 22.91 & 20.86 & 20.79 & 20.35 & 20.51 & 18.28 \\
\hline 247 & 540 & 27.89 & 26.15 & 25.48 & 25.37 & 24.57 & $\ldots$ & 21.82 & 21.29 & 20.81 & 20.34 & 19.19 & 17.04 \\
\hline 249 & 14708 & 25.31 & 24.40 & 23.24 & 22.54 & 21.67 & $\ldots$ & 20.49 & 19.95 & 20.06 & 20.30 & 20.32 & 18.31 \\
\hline 250 & 14158 & 26.64 & 25.32 & 25.01 & 24.60 & 24.06 & 23.71 & 23.46 & 22.57 & 22.29 & 21.94 & 21.89 & 22.16 \\
\hline 251 & 4302 & 24.35 & 22.89 & 22.41 & 22.43 & 21.74 & $\ldots$ & 21.40 & 21.23 & 21.44 & 21.17 & 20.82 & 18.95 \\
\hline 254 & 10429 & 26.47 & 25.05 & 25.18 & 25.18 & 24.49 & 24.08 & 23.74 & 23.21 & 23.15 & 22.85 & 22.79 & 21.04 \\
\hline 255 & 8374 & 26.07 & 25.80 & 25.08 & 24.50 & 22.88 & 22.34 & 22.01 & 21.32 & 21.23 & 21.29 & 21.64 & 20.35 \\
\hline 256 & 7822 & 22.42 & 22.91 & 22.55 & 22.50 & 22.09 & $\ldots$ & 21.47 & 20.75 & 20.53 & 20.28 & 20.27 & 18.61 \\
\hline 258 & 11006 & 25.20 & 24.66 & 24.52 & 24.44 & 24.33 & 23.85 & 23.59 & 21.93 & 21.70 & 21.17 & 20.78 & 18.78 \\
\hline
\end{tabular}


M. Brusa et al.: Black hole growth and starburst activity at $z=0.6-4$ in the Chandra Deep Field South, Online Material $p 7$

Table 2. continued.

\begin{tabular}{|c|c|c|c|c|c|c|c|c|c|c|c|c|c|}
\hline 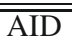 & OID & $\overline{\bar{b}}$ & $v$ & $i$ & $z$ & $j$ & $h$ & $\overline{k k}$ & 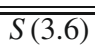 & $S(4.5)$ & $S(5.8)$ & $S(8.0)$ & $S(24)$ \\
\hline 259 & 1203 & 25.68 & 25.30 & 24.53 & 24.33 & 23.93 & $\ldots$ & 23.52 & 22.58 & 22.59 & 22.36 & 22.13 & 22.01 \\
\hline 260 & 4578 & 21.64 & 20.19 & 19.47 & 19.15 & 18.48 & $\ldots$ & 17.92 & 18.21 & 18.09 & 17.76 & 16.80 & 14.35 \\
\hline 261 & 3323 & 25.01 & 23.99 & 23.87 & 23.98 & 23.72 & $\ldots$ & 23.37 & 22.53 & 22.39 & 21.96 & 21.64 & 20.95 \\
\hline 262 & 8741 & 22.33 & 21.19 & 20.50 & 20.15 & 19.83 & 19.39 & 19.15 & 19.57 & 19.68 & 20.00 & 19.23 & 18.14 \\
\hline 263 & 7260 & 25.49 & 24.38 & 23.02 & 22.32 & 21.62 & $\ldots$ & 21.06 & 20.71 & 21.05 & 21.18 & 21.38 & 19.78 \\
\hline 264 & 8543 & 25.58 & 25.12 & 24.68 & 24.61 & 24.32 & 23.57 & 23.20 & 22.08 & 21.82 & 21.49 & 21.43 & 19.72 \\
\hline 266 & 9856 & 22.74 & 21.58 & 20.84 & 20.45 & 20.04 & 19.50 & 19.20 & 19.60 & 19.70 & 19.90 & 18.98 & 17.36 \\
\hline 268 & 11062 & 26.37 & 25.73 & 24.66 & 23.97 & 22.96 & 22.44 & 22.08 & 21.38 & 21.44 & 21.51 & 20.88 & 19.36 \\
\hline 269 & 1633 & 23.08 & 21.29 & 20.00 & 19.55 & 19.01 & $\ldots$ & 18.12 & 17.83 & 18.42 & 18.47 & 19.07 & 17.73 \\
\hline 272 & 2532 & 28.83 & 27.70 & 26.38 & 26.00 & 24.48 & $\ldots$ & 22.67 & 22.16 & 22.17 & 21.99 & 22.02 & 22.06 \\
\hline 273 & 4564 & 24.42 & 23.83 & 23.00 & 22.25 & 21.58 & $\ldots$ & 20.82 & 20.27 & 20.68 & 20.98 & 21.24 & 19.87 \\
\hline 276 & 3047 & 23.60 & 21.62 & 20.27 & 19.86 & 19.29 & $\ldots$ & 18.47 & 18.28 & 18.89 & 18.99 & 19.53 & 18.38 \\
\hline 277 & 1059 & 27.72 & 26.81 & 25.95 & 24.96 & 23.65 & $\ldots$ & 21.99 & 20.93 & 20.79 & 20.40 & 20.62 & $>21.53$ \\
\hline 281 & 3320 & 26.99 & 25.63 & 25.20 & 25.29 & 24.51 & .. & 23.03 & 23.48 & 22.27 & 22.61 & 20.44 & 18.50 \\
\hline 283 & 70435 & 28.44 & 28.00 & 27.26 & 26.88 & $>25.97$ & 24.06 & 23.95 & 22.51 & 22.18 & 21.63 & 21.30 & 19.25 \\
\hline 286 & 5062 & 23.64 & 22.86 & 22.26 & 21.80 & 21.30 & & 20.39 & 19.70 & 19.88 & 19.82 & 19.78 & 18.66 \\
\hline 292 & 2750 & 20.57 & 20.16 & 20.27 & 19.91 & 20.11 & $\ldots$ & 19.71 & 20.21 & 20.25 & 20.40 & 19.58 & 16.88 \\
\hline 300 & 4751 & 28.88 & 28.29 & 27.19 & 26.89 & 26.33 & $\ldots$ & 25.44 & 22.66 & $>24.92$ & 21.71 & 21.04 & $>20.69$ \\
\hline 70 & 70340 & 26.63 & 28.16 & 27.41 & $>27.56$ & 25.52 & $>25.46$ & $>25.62$ & 23.40 & 23.06 & 22.01 & 21.34 & 19.01 \\
\hline 81 & 6685 & 28.44 & 26.80 & 25.11 & 24.23 & 22.79 & 22.08 & 21.59 & 20.90 & 21.01 & 21.33 & 21.59 & 21.17 \\
\hline 89 & 70286 & 27.64 & 27.07 & 26.70 & 26.93 & 24.82 & $>24.88$ & 23.49 & 23.89 & 24.07 & $>23.30$ & $>23.03$ & 20.88 \\
\hline 104 & 70357 & $>28.83$ & 27.28 & 26.58 & 25.95 & 23.69 & 23.40 & 22.92 & 21.59 & 21.57 & 21.11 & 20.75 & 18.75 \\
\hline 128 & 70139 & 27.58 & 26.77 & 27.09 & 26.32 & 24.65 & 24.37 & 23.76 & 22.51 & 22.50 & 22.12 & 21.96 & 19.90 \\
\hline 135 & 80001 & 26.89 & 26.80 & 27.85 & 27.04 & 25.65 & 24.98 & $>25.35$ & 23.57 & 23.27 & 22.69 & 21.17 & 20.46 \\
\hline 140 & 70380 & 28.33 & $>28.72$ & $>27.94$ & $>27.66$ & 25.01 & 24.19 & 23.00 & 21.68 & 21.30 & 20.89 & 21.16 & $>21.02$ \\
\hline 144 & 70251 & 28.34 & 27.82 & 27.51 & 26.94 & $>25.88$ & 24.43 & 23.38 & 23.53 & 23.02 & 23.18 & 23.15 & $>21.02$ \\
\hline 232 & 70091 & $>28.60$ & 27.92 & 28.01 & 27.65 & 25.32 & 24.55 & 23.53 & 22.31 & 22.09 & 21.67 & 21.21 & 19.68 \\
\hline 74 & 11122 & 26.06 & 25.42 & 23.81 & 22.87 & 21.01 & 20.39 & 19.90 & 19.23 & 18.94 & 19.20 & 19.76 & $>21.09$ \\
\hline 85 & 16795 & 27.05 & 26.49 & 25.70 & 25.20 & 23.70 & & 22.83 & 21.97 & 21.76 & 21.95 & 21.96 & 21.30 \\
\hline 97 & 13053 & 27.98 & 25.11 & 23.68 & 23.25 & 22.76 & 22.26 & 21.94 & 22.25 & 22.48 & 22.62 & 23.03 & 20.76 \\
\hline 107 & 11065 & 23.94 & 23.62 & 23.09 & 22.89 & 22.43 & 22.46 & 22.17 & 22.37 & 21.33 & 22.91 & 22.48 & 19.38 \\
\hline 119 & 8977 & 25.60 & 24.29 & 23.06 & 22.54 & 21.66 & 20.94 & 20.43 & 20.01 & 20.48 & 20.45 & 20.74 & 18.63 \\
\hline 132 & 11695 & 21.44 & 20.65 & 20.31 & 20.21 & 20.06 & 19.88 & 19.72 & 20.41 & 20.60 & 20.95 & 20.23 & 19.76 \\
\hline 138 & 70198 & 28.34 & 28.89 & 26.58 & 26.22 & $>25.40$ & 23.28 & 23.53 & 21.17 & 21.37 & 20.94 & 20.99 & 18.42 \\
\hline 142 & 70307 & $>28.59$ & 27.24 & 27.89 & 26.43 & 24.19 & 23.66 & 22.75 & 21.75 & 21.80 & 21.12 & 20.71 & 19.50 \\
\hline 148 & 13552 & 22.51 & 21.61 & 20.62 & 20.29 & 19.71 & 19.21 & 18.72 & 18.70 & 19.10 & 19.14 & 19.38 & 17.01 \\
\hline 165 & 70449 & $>28.70$ & $>28.89$ & $>28.10$ & 27.70 & 24.82 & 23.57 & 22.70 & 21.30 & 20.88 & 20.37 & 20.22 & 18.54 \\
\hline 178 & 2768 & 24.35 & 23.78 & 22.92 & 22.22 & 21.63 & $\ldots$ & 20.65 & 20.04 & 20.70 & 20.84 & 21.19 & 19.73 \\
\hline 185 & 4402 & 22.90 & 22.54 & 22.22 & 22.21 & 22.19 & $\ldots$ & 21.97 & 22.33 & 22.72 & 23.00 & 22.97 & 21.21 \\
\hline 190 & 3290 & 26.85 & 25.80 & 24.46 & 23.62 & 22.80 & & 21.89 & 21.51 & 21.95 & 22.33 & 22.55 & $>21.49$ \\
\hline 199 & 13111 & 25.25 & 23.73 & 22.26 & 21.59 & 20.88 & 20.59 & 20.14 & 20.01 & 20.32 & 20.67 & 21.23 & 21.01 \\
\hline 203 & 17284 & 24.47 & 23.21 & 21.79 & 20.87 & 20.04 & 19.43 & 19.04 & 18.49 & 18.77 & 19.21 & 19.54 & 18.83 \\
\hline 215 & 7453 & 25.12 & 24.89 & 24.73 & 24.76 & 24.24 & 23.78 & 23.90 & 23.27 & 23.22 & 23.45 & $>24.46$ & 21.32 \\
\hline 217 & 70437 & $>28.69$ & 28.52 & 28.10 & 27.62 & $>26.02$ & $>25.35$ & 23.58 & 23.02 & 22.53 & 21.94 & 21.32 & 20.34 \\
\hline 225 & 7443 & 27.33 & 26.16 & 25.05 & 24.33 & 22.82 & 22.12 & 21.63 & 21.07 & 20.87 & 21.17 & 22.11 & $>21.14$ \\
\hline 239 & 3743 & 26.13 & 26.03 & 26.09 & 25.90 & 24.89 & $\ldots$ & 24.92 & 24.71 & 25.12 & 24.48 & $>23.57$ & $>21.51$ \\
\hline 248 & 9957 & 24.93 & 22.61 & 21.16 & 20.71 & 20.08 & 19.58 & 19.27 & 19.41 & 19.94 & 20.17 & 20.91 & 21.47 \\
\hline 257 & 8053 & 26.45 & 26.09 & 25.16 & 24.70 & 23.24 & $\ldots$ & 22.05 & 20.94 & 20.73 & 20.70 & 20.91 & 17.94 \\
\hline 265 & 1263 & 20.85 & 19.89 & 19.29 & 18.99 & 18.69 & $\ldots$ & 18.09 & 18.21 & 18.37 & 18.57 & 17.77 & 16.42 \\
\hline 267 & 564 & 20.80 & 20.48 & 20.26 & 20.36 & 20.32 & $\ldots$ & 20.22 & 20.83 & 21.17 & 21.27 & 20.65 & 17.79 \\
\hline 183 & 70241 & $>28.71$ & 27.11 & 26.37 & 25.79 & $>24.73$ & 23.25 & 22.76 & 21.85 & 21.94 & 21.57 & 21.40 & 20.07 \\
\hline 186 & 70403 & 27.25 & 27.83 & 26.67 & 26.56 & 25.27 & 24.77 & 24.28 & 22.31 & 22.43 & 21.95 & 21.95 & 19.98 \\
\hline 282 & $\ldots$ & $\ldots$ & $\ldots$ & $\ldots$ & $\ldots$ & $\ldots$ & $\ldots$ & $\ldots$ & $\ldots$ & $\ldots$ & $\ldots$ & $\ldots$ & $\ldots$ \\
\hline
\end{tabular}

Notes: all the magnitudes in the AB system. 